\title{
Viscosity Iterative Schemes for Finding Split Common Solutions of Variational Inequalities and Fixed Point Problems
}

\author{
Zhenhua He${ }^{1}$ and Wei-Shih $\mathrm{Du}^{2}$ \\ ${ }^{1}$ Department of Mathematics, Honghe University, Yunnan 661100, China \\ ${ }^{2}$ Department of Mathematics, National Kaohsiung Normal University, Kaohsiung 824, Taiwan \\ Correspondence should be addressed to Wei-Shih Du, wsdu@nknucc.nknu.edu.tw
}

Received 12 August 2012; Accepted 25 September 2012

Academic Editor: Yonghong Yao

Copyright (C) 2012 Z. He and W.-S. Du. This is an open access article distributed under the Creative Commons Attribution License, which permits unrestricted use, distribution, and reproduction in any medium, provided the original work is properly cited.

We introduce some new iterative schemes based on viscosity approximation method for finding a split common element of the solution set of a pair of simultaneous variational inequalities for inverse strongly monotone mappings in real Hilbert spaces with a family of infinitely nonexpansive mappings. Some strong convergence theorems are also given. Our results generalize and improve some well-known results in the literature and references therein.

\section{Introduction}

Throughout this paper, we denote by $\mathbb{N}$ and $\mathbb{R}$, the sets of positive integers and real numbers, respectively. Let $H$ be a real Hilbert space, whose inner product and norm are denoted by $\langle\cdot, \cdot\rangle$ and $\|\cdot\|$, respectively. Let $I$ be the identity mapping on $H$ and $C$ be a nonempty closed convex subset of $H$. Let $T: C \rightarrow H$ be a nonlinear operators. Then the canonical variational inequality problem for the operator $T\left((\mathrm{VIP})_{T}\right.$ or (VIP), for short) is to find $u \in C$ such that

$$
\langle T u, v-u\rangle \geq 0, \quad \forall v \in C
$$

We use the symbol $\mathrm{VI}(C, T)$ to denote the solution set of (VIP), that is

$$
\operatorname{VI}(C, T)=\{u \in C:\langle T u, v-u\rangle \geq 0, \forall v \in C\}
$$


(VIP) was extensively investigated and generalized to the vector variational inequality problems for single-valued or multivalued maps and contains optimization problems, quasivariational inequality problems, equilibrium problems, fixed-point problems, complementary problems, bilevel problems, and semi-infinite problems as special cases and applications; see [1-6] and references therein.

Let $S, T: C \rightarrow H$ be two nonlinear operators. In [7], some authors have considered the following pair of simultaneous variational inequality problems for operators $S$ and $T$ $\left((\text { PSVIP })_{S, T}\right.$, for short):

$$
(\mathrm{PSVIP})_{S, T} \text { Find } u \in C \text { such that }\langle S u, v-u\rangle \geq 0 \text { and }\langle T u, v-u\rangle \geq 0, \quad \forall v \in C .
$$

An element $u \in C$ is a solution of (PSVIP) $)_{S, T}$ if and only if $u \in \operatorname{VI}(C, S) \cap \operatorname{VI}(C, T)$. Clearly, $(\mathrm{PSVIP})_{S, T}$ reduces to (VIP) if $S=T$.

Example 1.1. Let $\mathbb{R}$ with usual inner product and let $a, b \in \mathbb{R}$ with $a<b$. Define two realvalued functions $T_{1}, T_{2}$ by $T_{1} x=x^{2}, T_{2} x=x^{4}$, for all $x \in[a, b]$. Then $T_{1}^{\prime} x=2 x, T_{2}^{\prime} x=4 x^{3}$ and there exists $x_{0} \in[a, b]$ such that $T_{1} x_{0}=\min _{x \in[a, b]} T_{1} x$ and $T_{2} x_{0}=\min _{x \in[a, b]} T_{2} x$. If $x_{0} \in(a, b)$, then $T_{1} x_{0}=T_{2} x_{0}=0$; if $x_{0}=a$, then $T_{1}^{\prime} x_{0} \geq 0$ and $T_{2}^{\prime} x_{0} \geq 0$; if $x_{0}=b$, then $T_{1}^{\prime} x_{0} \leq 0$ and $T_{2}^{\prime} x_{0} \leq 0$. So we have

$$
\left\langle T_{1}^{\prime} x_{0}, x-x_{0}\right\rangle=T_{1}^{\prime} x_{0}\left(x-x_{0}\right) \geq 0, \quad\left\langle T_{2}^{\prime} x_{0}, x-x_{0}\right\rangle=T_{2}^{\prime} x_{0}\left(x-x_{0}\right) \geq 0, \quad \forall x \in[a, b]
$$

or $x_{0} \in \mathrm{VI}\left(C, T_{1}^{\prime}\right) \cap \mathrm{VI}\left(C, T_{2}^{\prime}\right)$ which means that $x_{0}$ is the solution of (PSVIP) $)_{T_{1}^{\prime}, T_{2}^{\prime}}$.

Obviously, the problem (PSVIP) $)_{S, T}$ is considered in the same subset of the same space. But many cases, two variational inequality problems often lie in different subset of spaces. So, as a further development of the problem (PSVIP) $)_{S, T}$, Censor et al. [8] presented a split variational inequality problem. Let $H_{1}, H_{2}$ be two real Hilbert spaces and $C \subset H_{1}$ and $K \subset H_{2}$ two closed convex sets. Let $A: H_{1} \rightarrow H_{2}$ be a bounded linear operator. $T: C \rightarrow H_{1}$ and $S: K \rightarrow H_{2}$ are two nonlinear operators. The split variational inequality problem for $T$ and $S\left((\mathrm{SVIP})_{T, S}\right.$, for short) is defined as follows:

$$
\begin{aligned}
& (\mathrm{PSVIP})_{S, T} \text { Find } p \in C \text { such that }\langle T p, v-p\rangle \geq 0, \quad \forall v \in C, \\
& \text { and } u:=A p \in K \text { solves }\langle S u, w-u\rangle \geq 0, \quad \forall w \in K
\end{aligned}
$$

It is well known to find a solution of (VIP) or a common element of the solution set of (VIP) and a fixed point of nonlinear operators, which has been studied by many authors (see [9-16]) using all kinds of auxiliary techniques and formulations. In 2005, Iiduka and Takahashi [9] established the following iteration scheme: let $x_{1} \in H$ be arbitrary, define

$$
x_{n+1}=\alpha_{n} u+\left(1-\alpha_{n}\right) S_{1} P_{C}\left(x_{n}-\lambda_{n} T x_{n}\right),
$$


where $S_{1}$ is a nonexpansive mapping. They proved that the sequence $\left\{x_{n}\right\}$ defined by (1.6) strongly converge to $x^{*} \in F\left(S_{1}\right) \cap \operatorname{VI}(C, T)$, if the coefficient $\alpha_{n}, \lambda_{n}$ satisfy the following conditions:

$$
\lim _{n \rightarrow \infty} \alpha_{n}=0, \quad \sum_{n=1}^{\infty} \alpha_{n}=\infty, \quad \sum_{n=1}^{\infty}\left|\alpha_{n+1}-\alpha_{n}\right|<\infty, \quad \sum_{n=1}^{\infty}\left|\lambda_{n+1}-\lambda_{n}\right|<\infty .
$$

In 2007, Chen et al. [10] studied the following iterative process:

$$
x_{n+1}=\alpha_{n} f\left(x_{n}\right)+\left(1-\alpha_{n}\right) S_{1} P_{C}\left(x_{n}-\lambda_{n} T x_{n}\right),
$$

where $S_{1}$ is a nonexpansive mapping. If $\lim _{n \rightarrow \infty} \alpha_{n}=0, \sum_{n=1}^{\infty} \alpha_{n}=\infty, \sum_{n=1}^{\infty}\left|\alpha_{n+1}-\alpha_{n}\right|<$ $\infty$ and $\sum_{n=1}^{\infty}\left|\lambda_{n+1}-\lambda_{n}\right|<\infty$, then they proved that $\left\{x_{n}\right\}$ converges strongly to $q \in$ $F\left(S_{1}\right) \cap \operatorname{VI}(C, T)$, which solves the variational inequality:

$$
\langle f q-q, p-q\rangle \leq 0, \quad \forall p \in F\left(S_{1}\right) \cap \operatorname{VI}(C, T) .
$$

For some split common solution problems, they have been studied by some authors; see $[17,18]$ and therein references. In this paper, we continue to study the (SVIP) and introduce some new iterative schemes based on viscosity approximation method for finding a common element of the fixed points set of nonexpansive mappings and the split solution set of a pair of variational inequalities for inverse strongly monotone mappings in real Hilbert spaces. Our results are new development of finding a common element of fixed point of nonlinear operators and variational inequality problems.

\section{Preliminaries}

In this paper, we use symbols $\rightarrow$ and $\rightarrow$ to denote strong and weak convergence, respectively. A Banach space $(X,\|\cdot\|)$ is said to satisfy Opial's condition, if for each sequence $\left\{x_{n}\right\}$ in $X$ with $x_{n} \rightarrow x \in X$, we have

$$
\liminf _{n \rightarrow \infty}\left\|x_{n}-x\right\|<\liminf _{n \rightarrow \infty}\left\|x_{n}-y\right\|, \quad \forall y \in X, y \neq x
$$

It is well known that each Hilbert space satisfies Opial's condition; see, for example, [19]. Let $T: X \rightarrow X$ be a mapping. In this paper, the set of fixed points of $T$ is denoted by $F(T)$.

A set-valued mapping $T_{1}: H \rightarrow 2^{H}$ is said to be monotone, if for all $x, y \in H, f \in T_{1} x$, and $g \in T_{1} y$ imply that $\langle f-g, x-y\rangle \geq 0$. A monotone mapping $T_{1}: H \rightarrow H$ is said to be maximal, if the graph $G\left(T_{1}\right)$ of $T_{1}$ is not properly contained in the graph of any other monotone mapping. It is known that a monotone mapping $T_{1}$ is maximal, if and only if for $(x, f) \in H \times H,\langle f-g, x-y\rangle \geq 0$ for every $(y, g) \in G\left(T_{1}\right)$ implies that $f \in T_{1} x$. Let $T: C \rightarrow H$ be a monotone mapping and let $N_{C} v$ be the normal cone to $C$ at $v \in C$, that is, $N_{C} v=\{w \in$ $H:\langle v-u, w\rangle \geq 0$, for all $u \in C\}$. Define

$$
T_{1} v= \begin{cases}T v+N_{C} v, & v \in C \\ \emptyset, & v \notin C\end{cases}
$$


Then $T_{1}$ is maximal monotone and $\mathbf{0} \in T_{1} v$ if and only if $v \in \mathrm{VI}(C, T)$, where $\mathbf{0}$ is the zero vector of $H$; see, for example, $[9,20,21]$ for more details.

For any $x \in H$, there exists a unique nearest point in $C$, denoted by $P_{C}(x)$, such that $\left\|x-P_{C}(x)\right\| \leq\|x-y\|$ for all $y \in C$. The mapping $P_{C}$ is called the projection operator (or metric projection) from $H$ onto $C$.

Let $H_{1}$ and $H_{2}$ be two Hilbert spaces. Let $A: H_{1} \rightarrow H_{2}$ and $B: H_{2} \rightarrow H_{1}$ be two bounded linear operators. $B$ is called the adjoint operator (or adjoint) of $A$, if for all $z \in H_{1}, w \in H_{2}, B$ satisfies $\langle A z, w\rangle=\langle z, B w\rangle$. It is known that the adjoint operator of a bounded linear operator on a Hilbert space always exists and is bounded linear and unique. Moreover, it is not hard to show that if $B$ is an adjoint operator of $A$, then $\|A\|=\|B\|$.

A mapping $T: C \rightarrow C$ is said to be

(1) $v$-expansive if there exists a constant $v>0$ such that $\|T x-T y\| \geq v\|x-y\|$ for all $x, y \in C$. In particular, if $v=1$, then $T$ is called expansive.

(2) $v$-strongly monotone if there exists a constant $v>0$ such that

$$
\langle T x-T y, x-y\rangle \geq v\|x-y\|^{2}, \quad \forall x, y \in C
$$

Clearly, any $v$-strongly monotone mapping is $v$-expansive.

(3) $u$-inverse strongly monotone if there exists a constant $u>0$ such that

$$
\langle T x-T y, x-y\rangle \geq u\|T x-T y\|^{2}, \quad \forall x, y \in C
$$

(4) Relaxed $u$-cocoercive if there exists a constant $u>0$ such that

$$
\langle T x-T y, x-y\rangle \geq(-u)\|T x-T y\|^{2}, \quad \forall x, y \in C
$$

(5) Relaxed $(u, v)$-cocoercive if there exists constants $u, v>0$ such that

$$
\langle T x-T y, x-y\rangle \geq(-u)\|T x-T y\|^{2}+v\|x-y\|^{2}, \quad \forall x, y \in C .
$$

Especially, if $u=0$, then $T$ is $v$-strongly monotone. So this class of mapping is more general than the class of strongly monotone mapping.

(6) An $\alpha$-Lipschitz mapping if there exists a constant $\alpha>0$ such that $\|T x-T y\| \leq$ $\alpha\|x-y\|$ for all $x, y \in C$. In particular, if $0<\alpha<1(\alpha=1$, resp.), then $T$ is called a contraction (a nonexpansive mapping, resp.)

Remark ST (see [9]). If $T$ is $v$-strongly monotone and $\mu$-Lipschitz continuous, that is, $\| T x-$ $T y\|\leq \mu\| x-y \|$ for all $x, y \in C$, then $T$ is $\left(v / \mu^{2}\right)$-inverse strongly monotone.

Example 2.1. Let $T x=-2 x$, for all $x \in \mathbb{R}$. Then it is easy to see that for any $x, y \in \mathbb{R}$,

$$
\langle T x-T y, x-y\rangle=-2|x-y|^{2} \geq(-1)|T x-T y|^{2}+|x-y|^{2} .
$$

Hence $T$ is a relaxed $(1,1)$-cocoercive mapping, but $T$ is not a strongly monotone mapping. 
Now, let $\left\{T_{i}\right\}_{i \in \mathbb{N}}$ be a family of infinitely nonexpansive mappings. In [22], a mapping $W_{n}$ is defined by the following:

$$
\begin{aligned}
U_{n, n+1}= & I, \\
U_{n, n}= & \lambda_{n} T_{n} U_{n, n+1}+\left(1-\lambda_{n}\right) I, \\
U_{n, n-1}= & \lambda_{n-1} T_{n-1} U_{n, n}+\left(1-\lambda_{n-1}\right) I, \\
& \vdots \\
U_{n, k}= & \lambda_{k} T_{k} U_{n, k+1}+\left(1-\lambda_{k}\right) I, \\
U_{n, k-1}= & \lambda_{k-1} T_{k-1} U_{n, k}+\left(1-\lambda_{k-1}\right) I, \\
& \vdots \\
U_{n, 2}= & \lambda_{2} T_{2} U_{n, 3}+\left(1-\lambda_{2}\right) I, \\
W_{n}= & U_{n, 1}=\lambda_{1} T_{1} U_{n, 2}+\left(1-\lambda_{1}\right) I,
\end{aligned}
$$

where $\left\{\lambda_{i}\right\}_{i \in \mathbb{N}} \subset[0,1]$. Such a mapping $W_{n}$ is called the $W$-mapping generated by $T_{n}, T_{n-1}, \ldots, T_{1}$ and $\lambda_{n}, \lambda_{n-1}, \ldots, \lambda_{1}$.

The following properties for a $W$-mapping are well known.

Theorem 2.2 (see [22, 23]). Let $C$ be a nonempty closed convex subset of a Hilbert space E, let $T_{1}, T_{2}, \ldots$ be a family of infinitely nonexpansive mappings from $C$ into itself such that $\bigcap_{i=1}^{\infty} F\left(T_{i}\right)$ is nonempty, and let $\lambda_{1}, \lambda_{2}, \ldots$ be real numbers such that $0<\lambda_{i} \leq b<1$ for any $i \in \mathbb{N}$. Then the following statements hold:

(1) $W_{n}$ is a nonexpansive mapping and $F\left(W_{n}\right)=\bigcap_{i=1}^{n} F\left(T_{i}\right)$.

(2) For each $x \in C$ and for each positive integer $k$, the limit $\lim _{n \rightarrow \infty} U_{n, k} x$ exists.

(3) The mapping $W: C \rightarrow C$ defined by $W x:=\lim _{n \rightarrow \infty} W_{n} x=\lim _{n \rightarrow \infty} U_{n, 1} x, x \in C$, is a nonexpansive mapping satisfying $F(W)=\bigcap_{i=1}^{\infty} F\left(T_{i}\right)$ and it is called the $W$-mapping generated by $T_{1}, T_{2}, \ldots$ and $\lambda_{1}, \lambda_{2} \ldots$.

Theorem 2.3 (see [23]). Let $C$ be a nonempty closed convex subset of a Hilbert space $H, T_{1}, T_{2}, \ldots$ be nonexpansive mappings with $\bigcap_{i=1}^{\infty} F\left(T_{i}\right)=\emptyset,\left\{\lambda_{i}\right\}$ be a real sequence such that $0<\lambda_{i} \leq b<1$ for any $i \in \mathbb{N}$. If $K$ is any bounded subset of $C$, then

$$
\lim _{n \rightarrow \infty} \sup _{x \in K}\left\|W x-W_{n} x\right\|=0
$$

In particular, if $\left\{x_{n}\right\}_{n \in \mathbb{N}}$ is a bounded sequence in $C$, then $\lim _{n \rightarrow \infty}\left\|W x_{n}-W_{n} x_{n}\right\|=0$.

The following results are crucial in this paper.

Lemma 2.4 (see [19]). For a given $z \in H, x \in C$ satisfies the inequality $\langle x-z, y-x\rangle \geq$ 0 , for all $y \in C$ if and only if $x=P_{C}(z)$, where $P_{C}$ is a projection operator from $H$ onto $C$. 
It is well known that the projection operator $P_{C}$ is nonexpansive and satisfies

$$
\left\|P_{C} x-P_{C} y\right\|^{2} \leq\left\langle P_{C} x-P_{C} y, x-y\right\rangle, \quad \forall x, y \in H
$$

Lemma 2.5 (see [9]). The element $u \in C$ is a solution of $(V I P)_{T}$ if and only if $u \in C$ satisfies the relation $u=P_{C}(u-\rho T u)$, where $P_{C}$ is the projection operator, $\rho>0$ is a constant.

Lemma 2.6 (see [24]). Let $\left\{a_{n}\right\}$ be a nonnegative real sequence satisfying the following condition:

$$
a_{n+1} \leq\left(1-\lambda_{n}\right) a_{n}+\lambda_{n} b_{n}, \quad \forall n \geq n_{0},
$$

where $n_{0}$ is some nonnegative integer, $\left\{\lambda_{n}\right\}$ is a sequence in $(0,1)$ and $\left\{b_{n}\right\}$ is a sequence in $\mathbf{R}$ such that

(i) $\sum_{n=0}^{\infty} \lambda_{n}=\infty$;

(ii) $\lim \sup _{n \rightarrow \infty} b_{n} \leq 0$ or $\sum_{n=0}^{\infty} \lambda_{n} b_{n}$ is convergent.

Then $\lim _{n \rightarrow \infty} a_{n}=0$.

Lemma 2.7 (see [25]). Let $\left\{x_{n}\right\}$ and $\left\{y_{n}\right\}$ be bounded sequences in a Banach space $E$ and let $\left\{\beta_{n}\right\}$ be a sequence in $[0,1]$ with $0<\liminf _{n \rightarrow \infty} \beta_{n} \leq \limsup _{n \rightarrow \infty} \beta_{n}<1$. Suppose $x_{n+1}=\beta_{n} y_{n}+\left(1-\beta_{n}\right) x_{n}$ for all integers $n \geq 0$ and $\limsup _{n \rightarrow \infty}\left(\left\|y_{n+1}-y_{n}\right\|-\left\|x_{n+1}-x_{n}\right\|\right) \leq 0$, then $\lim _{n \rightarrow \infty}\left\|y_{n}-x_{n}\right\|=0$.

Lemma 2.8 (see [26]). Let $E$ be a real Banach space and $J: E \rightarrow 2^{E^{*}}$ be the normalized duality mapping, then for any $x, y \in E$ the following inequality holds:

$$
\|x+y\|^{2} \leq\|x\|^{2}+2\langle y, j(x+y)\rangle, \quad \forall j(x+y) \in J(x+y)
$$

Especially, when $E=H$, then $J=I$. So, from Lemma 2.8, one has

$$
\|x+y\|^{2} \leq\|x\|^{2}+2\langle y, x+y\rangle, \quad \forall x, y \in H
$$

The following result is simple, but it is very useful in this paper.

Lemma 2.9. Let $\left\{a_{n}\right\},\left\{b_{n}\right\}$ be two nonnegative real sequences. If $\lim _{n \rightarrow \infty} a_{n}=0$, then $\liminf _{n \rightarrow \infty}\left(a_{n}+b_{n}\right)=\liminf _{n \rightarrow \infty} b_{n}$.

\section{Main Results}

In this section, we construct an iteration scheme including a pair of mappings $T: C \rightarrow$ $H_{1}$ and $S: K \rightarrow H_{2}$ which are $u$-inverse strongly monotone to solve the split variational inequality problem. For the purpose we first give the following Lemmas.

Lemma 3.1 (see page 3 in [9]). Let $T: C \rightarrow H$ be a $u$-inverse strongly monotone mapping. Then $I-\lambda T$ is nonexpansive for any $\lambda \in[0,2 u]$. 
Example 3.2. Let $T x=3 x$ for all $x \in \mathbb{R}$ and $u=1 / 6$. Since

$$
\langle T x-T y, x-y\rangle=3|x-y|^{2} \geq u|T x-T y|^{2},
$$

$T$ is $u$-inversely monotone. Let $\lambda \in[0,1 / 3]=[0,2 u]$. It is easy to see that

$$
|(I-\lambda T) x-(I-\lambda T) y|=(1-3 \lambda)|x-y| \leq|x-y|
$$

So $I-\lambda T$ is nonexpansive for all $\lambda \in[0,2 u]$.

Applying Lemma 3.1, we have the following important result.

Lemma 3.3. Let $T, S: C \rightarrow H$ be two u-inverse strongly monotone mappings and $S_{1}: C \rightarrow C$ be a nonexpansive mapping. Then for any given sequences $\left\{r_{n}\right\}$ and $\left\{s_{n}\right\}$ in $[0,2 u], P_{C}\left(I-s_{n} T\right)$, $P_{C}\left(I-r_{n} S\right), S_{1} P_{C}\left(I-S_{n} T\right)$ and $S_{1} P_{C}\left(I-r_{n} S\right)$ are all nonexpansive for all $n \in \mathbb{N}$.

The following conclusion is immediate from Lemma 2.5 .

Lemma 3.4. The element $u \in C$ is a solution of $(S V I P)_{T, S}$ if and only if $u \in C$ satisfies the relation

$$
u=P_{C}(u-\rho T u), \quad A u=P_{K}(u-\rho S A u),
$$

where $P_{C}$ and $P_{K}$ are the projection operators, $\rho>0$ is a constant.

Theorem 3.5. Let $H_{1}, H_{2}$ be two real Hilbert spaces and $\mathrm{C} \subset H_{1}, K \subset H_{2}$ two nonempty closed convex sets. Let $T: C \rightarrow H_{1}$ and $S: K \rightarrow H_{2}$ be u-inversely monotone. Let $A: H_{1} \rightarrow H_{2}$ be a bounded linear operator with adjoint operator $A^{*}$. Let $f: C \rightarrow C$ be a contraction with contraction constant $\alpha$. Let $\left\{T_{i}\right\}_{i \in \mathbb{N}}$ be a family of infinitely nonexpansive mappings of $C$ into itself and $U$ a nonexpansive mapping of $K$ into itself such that $\Omega=\left\{p \in\left(\bigcap_{i=1}^{\infty} F\left(T_{i}\right)\right) \cap \operatorname{VI}(C, T): A p \in\right.$ $F(U) \cap \operatorname{VI}(K, S)\} \neq \emptyset$. Let $\xi$ be a real number and $\left\{\lambda_{i}\right\}_{i \in \mathbb{N}}$ be a sequence of real numbers such that $0<\lambda_{i} \leq \xi<1$ for every $i \in \mathbb{N}$. For each $n \in \mathbb{N}$, let $W_{n}$ be the $W$-mapping of $C$ into itself generated by $T_{n}, T_{n-1}, \ldots, T_{1}$ and $\lambda_{n}, \lambda_{n-1}, \ldots, \lambda_{1}$. Let $\left\{x_{n}\right\}$ be a sequence generated by the following algorithm:

$$
\begin{gathered}
x_{0}=x \in C \quad \text { chosen arbitrarily, } \\
y_{n}=P_{C}\left(I-\beta_{n} T\right) x_{n}, \\
l_{n}=P_{K}\left(I-\beta_{n} S\right) A y_{n}, \\
z_{n}=(1-\theta) x_{n}+\theta W_{n} P_{C}\left(y_{n}+r A^{*}\left(U l_{n}-A y_{n}\right)\right), \\
x_{n+1}=\alpha_{n} f\left(x_{n}\right)+\left(1-\alpha_{n}\right) z_{n}, \quad \forall n \in \mathbb{N} \cup\{0\},
\end{gathered}
$$

where $r \in\left(0,1 /\|A\|^{2}\right)$ and $\theta \in(0,1)$ are two constants and $\left\{\alpha_{n}\right\}_{n=0}^{\infty}$ and $\left\{\beta_{n}\right\}_{n=0}^{\infty}$ are two sequences in $(0,1)$. If $\left\{\alpha_{n}\right\}_{n=0}^{\infty}$ and $\left\{\beta_{n}\right\}_{n=0}^{\infty}$ further satisfy the following conditions:

$\left(C_{1}\right) \lim _{n \rightarrow \infty} \alpha_{n}=0$ and $\sum_{n=1}^{\infty} \alpha_{n}=\infty$,

$\left(C_{2}\right)\left\{\beta_{n}\right\} \subset[a, b]$ and $\lim _{n \rightarrow \infty}\left|\beta_{n+1}-\beta_{n}\right|=0$, where $0<a, b<2 u$, 
then the following statements hold:

(a) there exists a unique $q \in \Omega$ such that $P_{\Omega} f(q)=q$;

(b) $\left\{x_{n}\right\}$ converges strongly to $q$.

Proof. Let $p \in \Omega$. By Lemma 3.3, $P_{C}\left(I-\beta_{n} T\right)$, and $P_{K}\left(I-\beta_{n} S\right)$ are nonexpansive for all $n \in$ $\mathbb{N} \cup\{0\}$. For each $n \in \mathbb{N} \cup\{0\}$, by (3.4) and Lemma 3.4, we obtain the following inequalities:

$$
\begin{gathered}
\left\|y_{n}-p\right\|=\left\|P_{C}\left(I-\beta_{n} T\right) x_{n}-P_{C}\left(I-\beta_{n} T\right) p\right\| \leq\left\|x_{n}-p\right\|, \\
\left\|l_{n}-A p\right\|=\left\|P_{K}\left(I-\beta_{n} S\right) A y_{n}-P_{K}\left(I-\beta_{n} S\right) A p\right\| \leq\left\|A y_{n}-A p\right\|, \\
\left\|x_{n+1}-p\right\| \leq \alpha_{n}\left\|f\left(x_{n}\right)-p\right\|+\left(1-\alpha_{n}\right)\left\|z_{n}-p\right\| \leq \alpha_{n}\left\|f\left(x_{n}\right)-p\right\|+\left\|z_{n}-p\right\| .
\end{gathered}
$$

Let $h_{n}=P_{C}\left(y_{n}+r A^{*}\left(U l_{n}-A y_{n}\right)\right)$ for $n \in \mathbb{N} \cup\{0\}$. Then

$$
\begin{aligned}
\left\|W_{n} h_{n}-p\right\|^{2} \leq & \left\|h_{n}-p\right\|^{2}=\left\|P_{C}\left(y_{n}+r A^{*}\left(U l_{n}-A y_{n}\right)\right)-p\right\|^{2} \leq\left\|y_{n}+r A^{*}\left(U l_{n}-A y_{n}\right)-p\right\|^{2} \\
= & \left\|y_{n}-p\right\|^{2}+\left\|r A^{*}\left(U l_{n}-A y_{n}\right)\right\|^{2}+2 r\left\langle y_{n}-p, A^{*}\left(U l_{n}-A y_{n}\right)\right\rangle \\
\leq & \left\|y_{n}-p\right\|^{2}+\left\|r A^{*}\left(U l_{n}-A y_{n}\right)\right\|^{2} \\
& +2 r\left\langle A\left(y_{n}-p\right)+U l_{n}-A y_{n}-\left(U l_{n}-A y_{n}\right), U l_{n}-A y_{n}\right\rangle \\
= & \left\|y_{n}-p\right\|^{2}+\left\|r A^{*}\left(U l_{n}-A y_{n}\right)\right\|^{2} \\
& +2 r\left\{\frac{1}{2}\left\|U l_{n}-A p\right\|^{2}+\frac{1}{2}\left\|U l_{n}-A y_{n}\right\|^{2}-\left\|A y_{n}-A p\right\|^{2}-\left\|U l_{n}-A y_{n}\right\|^{2}\right\} \\
\leq & \left\|y_{n}-p\right\|^{2}+r^{2}\left\|A^{*}\right\|^{2}\left\|U l_{n}-A y_{n}\right\|^{2}-r\left\|U l_{n}-A y_{n}\right\|^{2} \\
= & \left\|y_{n}-p\right\|^{2}-r\left(1-r\left\|A^{*}\right\|^{2}\right)\left\|U l_{n}-A y_{n}\right\|^{2}, \\
\left\|z_{n}-p\right\|^{2}= & \left\|(1-\theta)\left(x_{n}-p\right)+\theta\left(W_{n} h_{n}-p\right)\right\|^{2} \\
& \leq(1-\theta)\left\|x_{n}-p\right\|^{2}+\theta\left\|W_{n} h_{n}-p\right\|^{2} \\
\leq & (1-\theta)\left\|x_{n}-p\right\|^{2}+\theta\left\|h_{n}-p\right\|^{2} \\
\leq & (1-\theta)\left\|x_{n}-p\right\|^{2}+\theta\left\|y_{n}-p\right\|^{2} \\
\leq & \left\|x_{n}-p\right\|^{2},
\end{aligned}
$$

for all $n \in \mathbb{N} \cup\{0\}$. Next, we will show that the conclusion is true by several steps.

Step 1. We show that all $\left\{x_{n}\right\},\left\{y_{n}\right\},\left\{z_{n}\right\},\left\{T x_{n}\right\},\left\{S A y_{n}\right\},\left\{l_{n}\right\}$, and $\left\{W_{n} h_{n}\right\}$ are bounded. 
To prove it, it suffices to show $\left\{x_{n}\right\}$ is bounded. Let $p \in \Omega$. We claim that

$$
\left\|x_{n}-p\right\| \leq \mathcal{L}:=\max \left\{\left\|x_{0}-p\right\|, \frac{\|f(p)-p\|}{1-\alpha}\right\} \quad \forall n \in \mathbb{N} \cup\{0\} .
$$

Indeed, it is obvious that (3.8) is true for $n=0$. Assume that (3.7) is true for $n=k, k \in \mathbb{N}$. Since $\left\|y_{k}-p\right\| \leq\left\|x_{k}-p\right\|$ and $\left\|z_{k}-p\right\| \leq\left\|x_{k}-p\right\|$ by (3.5) and (3.7), it follows from (3.5) that

$$
\begin{aligned}
\left\|x_{k+1}-p\right\| & \leq \alpha_{k}\left\|f\left(x_{k}\right)-p\right\|+\left(1-\alpha_{k}\right)\left\|z_{k}-p\right\| \\
& =\alpha_{k}\left\|f\left(x_{k}\right)-f\left(x_{k}\right)+f\left(x_{k}\right)-p\right\|+\left(1-\alpha_{k}\right)\left\|z_{k}-p\right\| \\
& \leq \alpha_{k} \alpha\left\|x_{k}-p\right\|+\alpha_{k}\|f(p)-p\|+\left(1-\alpha_{k}\right)\left\|x_{k}-p\right\| \\
& \leq\left(1-\alpha_{k}(1-\alpha)\right)\left\|x_{k}-p\right\|+\alpha_{k}\|f(p)-p\| \leq \mathcal{L}
\end{aligned}
$$

which prove that (3.8) is true for $n=k+1$. By induction, (3.8) holds for all $n \in \mathbb{N} \cup\{0\}$. Hence, by (3.8), we know that $\left\{x_{n}\right\}$ is bounded and so are $\left\{x_{n}\right\},\left\{y_{n}\right\},\left\{z_{n}\right\},\left\{T x_{n}\right\},\left\{S A y_{n}\right\},\left\{l_{n}\right\},\left\{h_{n}\right\}$, $\left\{W_{n} h_{n}\right\},\left\{l_{n}\right\}$, and $\left\{U l_{n}\right\}$. This also means that there exists a bounded subset $C_{1} \subset C$ such that

$$
\left\{x_{n}\right\},\left\{y_{n}\right\},\left\{z_{n}\right\},\left\{T x_{n}\right\},\left\{h_{n}\right\},\left\{W_{n} h_{n}\right\} \subset C_{1} .
$$

Step 2. Prove $\lim _{n \rightarrow \infty}\left\|x_{n+1}-x_{n}\right\|=0$.

For each $n \in \mathbb{N} \cup\{0\}$, by Lemma 3.1,

$$
\begin{aligned}
\left\|y_{n+1}-y_{n}\right\| & \leq\left\|P_{C}\left(I-\beta_{n+1} T\right) x_{n+1}-P_{C}\left(I-\beta_{n+1} T\right) x_{n}\right\|+\left\|P_{C}\left(I-\beta_{n+1} T\right) x_{n}-P_{C}\left(I-\beta_{n} T\right) x_{n}\right\| \\
& \leq\left\|x_{n+1}-x_{n}\right\|+\left|\beta_{n+1}-\beta_{n}\right|\left\|T x_{n}\right\| .
\end{aligned}
$$

Similarly,

$$
\begin{aligned}
\left\|l_{n+1}-l_{n}\right\| \leq & \left\|P_{K}\left(I-\beta_{n+1} S\right) A y_{n+1}-P_{K}\left(I-\beta_{n+1} S\right) A y_{n}\right\| \\
& +\left\|P_{K}\left(I-\beta_{n+1} S\right) A y_{n}-P_{K}\left(I-\beta_{n} S\right) A y_{n}\right\| \\
\leq & \left\|A y_{n+1}-A y_{n}\right\|+\left|\beta_{n+1}-\beta_{n}\right|\left\|S A y_{n}\right\|, \quad \forall n \in \mathbb{N} \cup\{0\} .
\end{aligned}
$$


Since $h_{n}=P_{C}\left(y_{n}+r A^{*}\left(U l_{n}-A y_{n}\right)\right), n \in \mathbb{N} \cup\{0\}$, we have

$$
\begin{aligned}
\left\|h_{n+1}-h_{n}\right\|^{2} \leq & \left\|y_{n+1}+r A^{*}\left(U l_{n+1}-A y_{n+1}\right)-\left(y_{n}+r A^{*}\left(U l_{n}-A y_{n}\right)\right)\right\|^{2} \\
\leq & \left\|y_{n+1}-y_{n}\right\|^{2}+\left\|r A^{*}\left(U l_{n+1}-A y_{n+1}-\left(U l_{n}-A y_{n}\right)\right)\right\|^{2} \\
& +2 r\left\langle y_{n+1}-y_{n}, A^{*}\left(U l_{n+1}-A y_{n+1}-\left(U l_{n}-A y_{n}\right)\right)\right\rangle \\
\leq & \left\|y_{n+1}-y_{n}\right\|^{2}+r^{2}\left\|A^{*}\right\|^{2}\left\|U l_{n+1}-A y_{n+1}-\left(U l_{n}-A y_{n}\right)\right\|^{2} \\
& +2 r\left\langle A y_{n+1}-A y_{n}+U l_{n+1}-A y_{n+1}-\left(U l_{n}-A y_{n}\right), U l_{n+1}-A y_{n+1}-\left(U l_{n}-A y_{n}\right)\right\rangle \\
& -2 r\left\langle U l_{n+1}-A y_{n+1}-\left(U l_{n}-A y_{n}\right), U l_{n+1}-A y_{n+1}-\left(U l_{n}-A y_{n}\right)\right\rangle \\
= & \left\|y_{n+1}-y_{n}\right\|^{2}+r^{2}\left\|A^{*}\right\|^{2}\left\|U l_{n+1}-A y_{n+1}-\left(U l_{n}-A y_{n}\right)\right\|^{2} \\
& +2 r\left\{\frac{1}{2} \mid U l_{n+1}-U l_{n}\left\|^{2}+\frac{1}{2}\right\| U l_{n+1}-A y_{n+1}-\left(U l_{n}-A y_{n}\right)\left\|-\frac{1}{2}\right\| A y_{n+1}-A y_{n} \|^{2}\right\} \\
& -2 r\left\|U l_{n+1}-A y_{n+1}-\left(U l_{n}-A y_{n}\right)\right\|^{2} \\
= & \left\|y_{n+1}-y_{n}\right\|^{2}-r\left(1-r\left\|A^{*}\right\|^{2}\right)\left\|U l_{n+1}-A y_{n+1}-\left(U l_{n}-A y_{n}\right)\right\|^{2} \\
& +r\left\{\left\|U l_{n+1}-U l_{n}\right\|^{2}-\left\|A y_{n+1}-A y_{n}\right\|^{2}\right\} \\
= & \left\|y_{n+1}-y_{n}\right\|^{2}-r\left(1-r\left\|A^{*}\right\|^{2}\right)\left\|U l_{n+1}-A y_{n+1}-\left(U l_{n}-A y_{n}\right)\right\|^{2} \\
& +r\left|\beta_{n+1}-\beta_{n}\right|\left(\left\|U l_{n+1}-U l_{n}\right\|+\left\|A y_{n+1}-A y_{n}\right\|\right)\left\|S A y_{n}\right\| \\
\leq & \left\|x_{n+1}-x_{n}\right\|^{2}+\left|\beta_{n+1}-\beta_{n}\right|\left(\left\|T x_{n}\right\|\left\|x_{n+1}-x_{n}\right\|+\left|\beta_{n+1}-\beta_{n}\right|\left\|T x_{n}\right\|^{2}\right) \\
& -r\left(1-r\left\|A^{*}\right\|^{2}\right)\left\|U l_{n+1}-A y_{n+1}-\left(U l_{n}-A y_{n}\right)\right\|^{2} \\
& +r\left|\beta_{n+1}-\beta_{n}\right|\left(\left\|U l_{n+1}-U l_{n}\right\|+\left\|A y_{n+1}-A y_{n}\right\|\right)\left\|S A y_{n}\right\| \\
\leq & \left\|x_{n+1}-x_{n}\right\|^{2}+\left|\beta_{n+1}-\beta_{n}\right| M_{1}, \\
& \left.+U l^{2}\right) \\
&
\end{aligned}
$$

where $M_{1}$ is a constant such that

$$
\left(\left\|T x_{n}\right\|\left\|x_{n+1}-x_{n}\right\|+\left|\beta_{n+1}-\beta_{n}\right|\left\|T x_{n}\right\|^{2}\right)+r\left(\left\|U l_{n+1}-U l_{n}\right\|+\left\|A y_{n+1}-A y_{n}\right\|\right)\left\|S A y_{n}\right\| \leq M_{1}
$$


for any $n \in \mathbb{N} \cup\{0\}$. Since $\left\{h_{n}\right\} \subset C_{1}$, for each $n \in \mathbb{N} \cup\{0\}$, we have

$$
\begin{aligned}
\left\|W_{n+1} h_{n+1}-W_{n} h_{n}\right\| \leq & \left\|W_{n+1} h_{n+1}-W h_{n+1}\right\|+\left\|W h_{n+1}-W h_{n}\right\|+\left\|W h_{n}-W_{n} h_{n}\right\| \\
& \leq \sup _{x \in C_{1}}\left\|W_{n+1} x-W x\right\|+\sup _{x \in C_{1}}\left\|W x-W_{n} x\right\|+\left\|h_{n+1}-h_{n}\right\|, \\
& \left\|W_{n+1} h_{n+1}-W_{n} h_{n}\right\|^{2} \leq\left\|h_{n+1}-h_{n}\right\|^{2}+\omega_{n},
\end{aligned}
$$

where

$$
\begin{aligned}
\omega_{n}= & \left(\sup _{x \in C_{1}}\left\|W_{n+1} x-W x\right\|+\sup _{x \in C_{1}}\left\|W x-W_{n} x\right\|\right) \\
& \times\left(\sup _{x \in C_{1}}\left\|W_{n+1} x-W x\right\|+\sup _{x \in C_{1}}\left\|W x-W_{n} x\right\|+2\left\|h_{n+1}-h_{n}\right\|\right) .
\end{aligned}
$$

So, we have

$$
\begin{aligned}
\left\|z_{n+1}-z_{n}\right\|^{2} & \leq(1-\theta)\left\|x_{n+1}-x_{n}\right\|^{2}+\theta\left\|W_{n+1} h_{n+1}-W_{n} h_{n}\right\|^{2} \\
& \leq(1-\theta)\left\|x_{n+1}-x_{n}\right\|^{2}+\theta\left\|h_{n+1}-h_{n}\right\|^{2}+\omega_{n} \\
& \leq(1-\theta)\left\|x_{n+1}-x_{n}\right\|^{2}+\theta\left\|x_{n+1}-x_{n}\right\|^{2}+\left|\beta_{n+1}-\beta_{n}\right| M_{1}+\omega_{n} \\
& =\left\|x_{n+1}-x_{n}\right\|^{2}+\left|\beta_{n+1}-\beta_{n}\right| M_{1}+\omega_{n},
\end{aligned}
$$

for any $n \in \mathbb{N} \cup\{0\}$.

Choose a sequence $\left\{\bar{y}_{n}\right\}$ such that $x_{n+1}=\gamma_{n} \bar{y}_{n}+\left(1-\gamma_{n}\right) x_{n}$, where $\gamma_{n}=1-(1-\theta)\left(1-\alpha_{n}\right)$, then we have

$$
\bar{y}_{n}=\frac{\alpha_{n} f\left(x_{n}\right)+\left(1-\alpha_{n}\right) \theta W_{n} h_{n}}{\gamma_{n}}, \quad \forall n \in \mathbb{N} \cup\{0\} .
$$

It follows that

$$
\begin{aligned}
\left\|\bar{y}_{n+1}-\bar{y}_{n}\right\| \leq & \frac{\alpha_{n}}{\gamma_{n}}\left\|f\left(x_{n}\right)\right\|+\frac{\alpha_{n+1}}{\gamma_{n+1}}\left\|f\left(x_{n+1}\right)\right\| \\
& +\frac{\left(1-\alpha_{n+1}\right) \theta}{\gamma_{n+1}}\left\|W_{n+1} h_{n+1}-W_{n} h_{n}\right\|+\left|\frac{\left(1-\alpha_{n+1}\right) \theta}{\gamma_{n+1}}-\frac{\left(1-\alpha_{n}\right) \theta}{\gamma_{n}}\right|\left\|W_{n} h_{n}\right\| \\
\leq & \left(\alpha_{n}+\alpha_{n+1}\right) M_{3}+\frac{\left(1-\alpha_{n+1}\right) \theta}{\gamma_{n+1}}\left\|W_{n+1} h_{n+1}-W_{n} h_{n}\right\|+\left|\frac{\alpha_{n}-\alpha_{n+1}}{\gamma_{n+1} \gamma_{n}}\right| \theta\left\|W_{n} h_{n}\right\| \\
\leq & 2\left(\alpha_{n}+\alpha_{n+1}\right) M_{3}+\frac{\left(1-\alpha_{n+1}\right) \theta}{\gamma_{n+1}}\left\|W_{n+1} h_{n+1}-W_{n} h_{n}\right\|,
\end{aligned}
$$


where $M_{3}$ is a constant such that $\sup _{n \in \mathbb{N} \cup\{0\}}\left\{\left\|f\left(x_{n}\right) / \gamma_{n}\right\|,\left\|W_{n} h_{n}\right\|\right\} \leq M_{3}$. From (3.20), (3.16), and (3.13) we have

$$
\begin{aligned}
\left\|\bar{y}_{n+1}-\bar{y}_{n}\right\|^{2} \leq & 4\left(\alpha_{n+1}+\alpha_{n}\right)^{2} M_{3}^{2}+\frac{\left(1-\alpha_{n+1}\right)^{2} \theta^{2}}{r_{n+1}^{2}}\left\|W_{n+1} h_{n+1}-W_{n} h_{n}\right\|^{2} \\
& +4\left(\alpha_{n}+\alpha_{n+1}\right) M_{3} \frac{\left(1-\alpha_{n+1}\right) \theta}{r_{n+1}}\left\|W_{n+1} h_{n+1}-W_{n} h_{n}\right\| \\
\leq & 4\left(\alpha_{n+1}+\alpha_{n}\right)^{2} M_{3}^{2}+\frac{\left(1-\alpha_{n+1}\right)^{2} \theta^{2}}{r_{n+1}^{2}}\left\|h_{n+1}-h_{n}\right\|^{2}+\frac{\left(1-\alpha_{n+1}\right)^{2} \theta^{2}}{r_{n+1}^{2}} \omega_{n} \\
& +4\left(\alpha_{n}+\alpha_{n+1}\right) M_{3} \frac{\left(1-\alpha_{n+1}\right) \theta}{\gamma_{n+1}}\left\|W_{n+1} h_{n+1}-W_{n} h_{n}\right\| \\
\leq & 4\left(\alpha_{n+1}+\alpha_{n}\right)^{2} M_{3}^{2}+\frac{\left(1-\alpha_{n+1}\right)^{2} \theta^{2}}{r_{n+1}^{2}}\left\|x_{n+1}-x_{n}\right\|^{2}+\frac{\left(1-\alpha_{n+1}\right)^{2} \theta^{2}}{r_{n+1}^{2}}\left|\beta_{n+1}-\beta_{n}\right| M_{1} \\
& +\frac{\left(1-\alpha_{n+1}\right)^{2} \theta^{2}}{r_{n+1}^{2}} \omega_{n}+4\left(\alpha_{n}+\alpha_{n+1}\right) M_{3} \frac{\left(1-\alpha_{n+1}\right) \theta}{\gamma_{n+1}}\left\|W_{n+1} h_{n+1}-W_{n} h_{n}\right\| .
\end{aligned}
$$

Applying the condition (C2), it follows from (3.21) that

$$
\limsup _{n \rightarrow \infty}\left\{\left\|\bar{y}_{n+1}-\bar{y}_{n}\right\|^{2}-\left\|x_{n+1}-x_{n}\right\|^{2}\right\}=0
$$

which implies

$$
\limsup _{n \rightarrow \infty}\left\{\left\|\bar{y}_{n+1}-\bar{y}_{n}\right\|-\left\|x_{n+1}-x_{n}\right\|\right\}=0 .
$$

Applying Lemma 2.7, we obtain $\lim _{n \rightarrow \infty}\left\|\bar{y}_{n}-x_{n}\right\| \rightarrow 0$ which implies that

$$
\lim _{n \rightarrow \infty}\left\|x_{n+1}-x_{n}\right\|=\lim _{n \rightarrow \infty} \gamma_{n}\left\|\bar{y}_{n}-x_{n}\right\|=0
$$

Step 3. Prove $\lim _{n \rightarrow \infty}\left\|T x_{n}-T p\right\|=\lim _{n \rightarrow \infty}\left\|S A y_{n}-S A p\right\|=0$.

For any $n \in \mathbb{N} \cup\{0\}$, we have

$$
\begin{aligned}
\left\|y_{n}-p\right\|^{2} & =\left\|P_{C}\left(I-\beta_{n} T\right) x_{n}-P_{C}\left(I-\beta_{n} T\right) p\right\|^{2} \\
& \leq\left\|\left(I-\beta_{n} T\right) x_{n}-\left(I-\beta_{n} T\right) p\right\|^{2} \\
& =\left\|x_{n}-p\right\|^{2}-2 \beta_{n}\left\langle x_{n}-p, T x_{n}-T p\right\rangle+\beta_{n}^{2}\left\|T x_{n}-T p\right\|^{2} \\
& \leq\left\|x_{n}-p\right\|^{2}-\beta_{n}\left(2 u-\beta_{n}\right)\left\|T x_{n}-T p\right\|^{2} .
\end{aligned}
$$


Similarly,

$$
\begin{aligned}
\left\|U l_{n}-A p\right\|^{2} & \leq\left\|l_{n}-A p\right\|^{2}=\left\|P_{K}\left(I-\beta_{n} S\right) A y_{n}-A p\right\|^{2} \\
& \leq\left\|A y_{n}-A p\right\|^{2}-\beta_{n}\left(2 u-\beta_{n}\right)\left\|S A y_{n}-S A p\right\|^{2}
\end{aligned}
$$

From (3.5) again, we have

$$
\left\|x_{n+1}-p\right\|^{2} \leq\left(\alpha_{n}\left\|f\left(x_{n}\right)-p\right\|+\left\|z_{n}-p\right\|\right)^{2} \leq \alpha_{n} M_{4}+\left\|z_{n}-p\right\|^{2},
$$

where $M_{4}$ is a constant such that $\sup _{n \in \mathbb{N} \cup\{0\}}\left\{\alpha_{n}\left\|f\left(x_{n}\right)-p\right\|^{2}+2\left\|f\left(x_{n}\right)-p\right\|\left\|z_{n}-p\right\|\right\} \leq M_{4}$. It follows that

$$
\begin{aligned}
0<\theta \beta_{n}\left(2 u-\beta_{n}\right)\left\|T x_{n}-T p\right\|^{2} \leq & \theta\left\|x_{n}-p\right\|^{2}-\theta\left\|y_{n}-p\right\|^{2} \quad(\text { by (3.24) }) \\
\leq & \left\|x_{n}-p\right\|^{2}-\left\|z_{n}-p\right\|^{2} \quad(\text { by (3.7) }) \\
\leq & \left\|x_{n}-p\right\|^{2}+\alpha_{n} M_{4}-\left\|x_{n+1}-p\right\|^{2} \quad(\text { by (3.26)) } \\
\leq & \left(\left\|x_{n}-p\right\|+\left\|x_{n+1}-p\right\|\right)\left\|x_{n+1}-x_{n}\right\|+\alpha_{n} M_{4} \\
& \longrightarrow 0 \quad \text { as } n \longrightarrow \infty,
\end{aligned}
$$

which yields that $\lim _{n \rightarrow \infty}\left\|T x_{n}-T p\right\|=0$ (by the condition $0<a \leq \beta_{n} \leq b<2 u$ ).

For any $n \in \mathbb{N} \cup\{0\}$, by (3.6), (3.7), and (3.27), we have

$$
\begin{aligned}
\theta r\left(1-r\left\|A^{*}\right\|^{2}\right)\left\|U l_{n}-A y_{n}\right\|^{2} & \leq \theta\left\|y_{n}-p\right\|^{2}-\theta\left\|W_{n} h_{n}-p\right\|^{2} \\
& \leq \theta\left\|x_{n}-p\right\|^{2}-\theta\left\|z_{n}-p\right\|^{2} \\
& \leq \theta\left\|x_{n}-p\right\|^{2}+\alpha_{n} M_{4}-\left\|x_{n+1}-p\right\|^{2} \\
& =\left(\left\|x_{n}-p\right\|+\left\|x_{n+1}-p\right\|\right)\left\|x_{n+1}-x_{n}\right\|+\alpha_{n} M_{4} .
\end{aligned}
$$

So,

$$
\lim _{n \rightarrow \infty}\left\|U l_{n}-A y_{n}\right\|=0
$$

From (3.26) and (3.30) again, we have

$$
\begin{aligned}
0<\beta_{n}\left(2 u-\beta_{n}\right)\left\|S A y_{n}-S A p\right\|^{2} \leq & \left\|A y_{n}-A p\right\|^{2}-\left\|U l_{n}-A p\right\|^{2} \\
= & \left(\left\|A y_{n}-A p\right\|+\left\|U l_{n}-A p\right\|\right)\left(\left\|A y_{n}-A p\right\|-\left\|U l_{n}-A p\right\|\right) \\
\leq & \left(\left\|A y_{n}-A p\right\|+\|A p\|\right)\left\|U l_{n}-A y_{n}\right\| \\
& \longrightarrow 0 \quad \text { as } n \longrightarrow \infty,
\end{aligned}
$$


which implies that $\lim _{n \rightarrow \infty}\left\|S A y_{n}-S A p\right\|=0$ (by the condition $0<a \leq \beta_{n} \leq b<2 u$ ).

On the other hand, since

$$
\begin{aligned}
\left\|l_{n}-A p\right\|^{2}= & \left\|P_{K}\left(I-\beta_{n} S\right) A y_{n}-P_{K}\left(I-\beta_{n} S\right) A p\right\|^{2} \\
\leq & \left\langle\left(I-\beta_{n} S\right) A y_{n}-\left(I-\beta_{n} S\right) A p, I_{n}-A p\right\rangle \\
= & \frac{1}{2}\left\{\left\|\left(I-\beta_{n} S\right) A y_{n}-\left(I-\beta_{n} S\right) A p\right\|^{2}\right. \\
& \left.\quad+\left\|l_{n}-A p\right\|^{2}-\left\|l_{n}-A y_{n}-\beta_{n}\left(S A y_{n}-S A p\right)\right\|^{2}\right\} \\
\leq & \frac{1}{2}\left\{\left\|A y_{n}-A p\right\|^{2}+\left\|l_{n}-A p\right\|^{2}-\left\|l_{n}-A y_{n}\right\|^{2}\right. \\
\quad & \left.\quad+2 \beta_{n}\left\langle l_{n}-A y_{n}, S A y_{n}-S A p\right\rangle-\beta_{n}^{2}\left\|S A y_{n}-S A p\right\|^{2}\right\} \\
\leq & \frac{1}{2}\left\{\left\|A y_{n}-A p\right\|^{2}+\left\|l_{n}-A p\right\|^{2}-\left\|l_{n}-A y_{n}\right\|^{2}+2 \beta_{n}\left\|l_{n}-A y_{n}\right\|\left\|S A y_{n}-S A p\right\|\right\},
\end{aligned}
$$

we get

$$
\left\|l_{n}-A p\right\|^{2} \leq\left\|A y_{n}-A p\right\|^{2}-\left\|l_{n}-A y_{n}\right\|^{2}+2 \beta_{n}\left\|l_{n}-A y_{n}\right\|\left\|S A y_{n}-S A p\right\|, \quad \forall n \in \mathbb{N} \cup\{0\}
$$

By (3.6) and (3.33), we have

$$
\begin{aligned}
\left\|W_{n} h_{n}-p\right\|^{2} \leq & \left\|y_{n}-p\right\|^{2}+\left\|r A^{*}\left(U l_{n}-A y_{n}\right)\right\|^{2} \\
& +2 r\left\{\frac{1}{2}\left\|U l_{n}-A p\right\|^{2}+\frac{1}{2}\left\|U l_{n}-A y_{n}\right\|^{2}-\left\|A y_{n}-A p\right\|^{2}-\left\|U l_{n}-A y_{n}\right\|^{2}\right\} \\
\leq & \left\|y_{n}-p\right\|^{2}+\left\|r A^{*}\left(U l_{n}-A y_{n}\right)\right\|^{2} \\
& +2 r\left\{\frac{1}{2}\left\|l_{n}-A p\right\|^{2}+\frac{1}{2}\left\|U l_{n}-A y_{n}\right\|^{2}-\left\|A y_{n}-A p\right\|^{2}-\left\|U l_{n}-A y_{n}\right\|^{2}\right\} \\
\leq & \left\|y_{n}-p\right\|^{2}+\left\|r A^{*}\left(U l_{n}-A y_{n}\right)\right\|^{2} \\
& +2 r\left\{-\frac{1}{2}\left\|l_{n}-A y_{n}\right\|^{2}+\beta_{n}\left\|l_{n}-A y_{n}\right\|\left\|S A y_{n}-S A p\right\|-\frac{1}{2}\left\|U l_{n}-A y_{n}\right\|^{2}\right\} \\
\leq & \left\|y_{n}-p\right\|^{2}+r^{2}\left\|A^{*}\right\|^{2}\left\|U l_{n}-A y_{n}\right\|^{2}-r\left\|U l_{n}-A y_{n}\right\|^{2}-r\left\|l_{n}-A y_{n}\right\|^{2} \\
& +2 r \beta_{n}\left\|l_{n}-A y_{n}\right\|\left\|S A y_{n}-S A p\right\| \\
= & \left\|y_{n}-p\right\|^{2}+r\left(1-r\left\|A^{*}\right\|^{2}\right)\left\|U l_{n}-A y_{n}\right\|^{2}-r\left\|l_{n}-A y_{n}\right\|^{2} \\
& +2 r \beta_{n}\left\|l_{n}-A y_{n}\right\|\left\|S A y_{n}-S A p\right\| \\
\leq & \left\|y_{n}-p\right\|^{2}-r\left\|l_{n}-A y_{n}\right\|^{2}+2 r \beta_{n}\left\|l_{n}-A y_{n}\right\|\left\|S A y_{n}-S A p\right\|,
\end{aligned}
$$


Using (3.7), (3.27), and (3.34), we obtain

$$
\begin{aligned}
\theta r\left\|l_{n}-A y_{n}\right\|^{2} & \leq \theta\left\|y_{n}-p\right\|^{2}-\theta\left\|W_{n} h_{n}-p\right\|^{2}+2 r \theta \beta_{n}\left\|l_{n}-A y_{n}\right\|\left\|S A y_{n}-S A p\right\| \\
& \leq \theta\left\|x_{n}-p\right\|^{2}-\theta\left\|z_{n}-p\right\|^{2}+2 r \theta \beta_{n}\left\|l_{n}-A y_{n}\right\|\left\|S A y_{n}-S A p\right\| \\
\leq & \theta\left\|x_{n}-p\right\|^{2}+\alpha_{n} M_{4}-\left\|x_{n+1}-p\right\|^{2}+2 r \theta \beta_{n}\left\|l_{n}-A y_{n}\right\|\left\|S A y_{n}-S A p\right\| \\
& =\left(\left\|x_{n}-p\right\|+\left\|x_{n+1}-p\right\|\right)\left\|x_{n+1}-x_{n}\right\|+\alpha_{n} M_{4}+2 r \theta \beta_{n}\left\|l_{n}-A y_{n}\right\|\left\|S A y_{n}-S A p\right\| \\
& \longrightarrow 0 \text { as } n \longrightarrow \infty,
\end{aligned}
$$

which implies

$$
\lim _{n \rightarrow \infty}\left\|l_{n}-A y_{n}\right\|=0
$$

According to (3.30) and (3.36), we derive that

$$
\lim _{n \rightarrow \infty}\left\|U l_{n}-l_{n}\right\|=0
$$

Step 4. Prove $\lim _{n \rightarrow \infty}\left\|x_{n}-z_{n}\right\|=\lim _{n \rightarrow \infty}\left\|y_{n}-x_{n}\right\|=\lim _{n \rightarrow \infty}\left\|h_{n}-x_{n}\right\|=0$.

Since

$$
\lim _{n \rightarrow \infty}\left\|x_{n+1}-x_{n}\right\|=0, \quad \lim _{n \rightarrow \infty}\left\|x_{n+1}-z_{n}\right\|=\lim _{n \rightarrow \infty} \alpha_{n}\left\|f\left(x_{n}\right)-z_{n}\right\|=0,
$$

we have $\lim _{n \rightarrow \infty}\left\|x_{n}-z_{n}\right\|=0$. For any $n \in \mathbb{N} \cup\{0\}$, since

$$
\begin{aligned}
\left\|y_{n}-p\right\|^{2} & =\left\|P_{C}\left(I-\beta_{n} T\right) x_{n}-P_{C}\left(I-\beta_{n} T\right) p\right\|^{2} \\
& \leq\left\langle\left(I-\beta_{n} T\right) x_{n}-\left(I-\beta_{n} T\right) p, y_{n}-p\right\rangle \\
& =\frac{1}{2}\left\{\left\|\left(I-\beta_{n} T\right) x_{n}-\left(I-\beta_{n} T\right) p\right\|^{2}+\left\|y_{n}-p\right\|^{2}-\left\|x_{n}-y_{n}-\beta_{n}\left(T x_{n}-T p\right)\right\|^{2}\right\} \\
& \leq \frac{1}{2}\left\{\left\|x_{n}-p\right\|^{2}+\left\|y_{n}-p\right\|^{2}-\left\|x_{n}-y_{n}\right\|^{2}+2 \beta_{n}\left\langle x_{n}-y_{n}, T x_{n}-T p\right\rangle-\beta_{n}^{2}\left\|T x_{n}-T p\right\|^{2}\right\} \\
& \leq \frac{1}{2}\left\{\left\|x_{n}-p\right\|^{2}+\left\|y_{n}-p\right\|^{2}-\left\|x_{n}-y_{n}\right\|^{2}+2 \beta_{n}\left\|x_{n}-y_{n}\right\|\left\|T x_{n}-T p\right\|\right\},
\end{aligned}
$$

we get

$$
\left\|y_{n}-p\right\|^{2} \leq\left\|x_{n}-p\right\|^{2}-\left\|x_{n}-y_{n}\right\|^{2}+2 \beta_{n}\left\|x_{n}-y_{n}\right\|\left\|T x_{n}-T p\right\| .
$$


It follows from (3.27), (3.7), and (3.40) that

$$
\begin{aligned}
\left\|x_{n+1}-p\right\|^{2} & \leq \alpha_{n} M_{4}+\left\|z_{n}-p\right\|^{2} \\
& \leq \alpha_{n} M_{4}+(1-\theta)\left\|x_{n}-p\right\|^{2}+\theta\left\|y_{n}-p\right\|^{2} \\
& \leq \alpha_{n} M_{4}+\left\|x_{n}-p\right\|^{2}-\theta\left\|x_{n}-y_{n}\right\|^{2}+2 \theta \beta_{n}\left\|x_{n}-y_{n}\right\|\left\|T x_{n}-T p\right\|,
\end{aligned}
$$

which yields that

$$
\begin{aligned}
\theta\left\|x_{n}-y_{n}\right\|^{2} & \leq \alpha_{n} M_{4}+\left\|x_{n}-p\right\|^{2}-\left\|x_{n+1}-p\right\|^{2}+2 \beta_{n}\left\|x_{n}-y_{n}\right\|\left\|T x_{n}-T p\right\| \\
& \leq \alpha_{n} M_{4}+\left(\left\|x_{n}-p\right\|+\left\|x_{n+1}-p\right\|\right)\left\|x_{n+1}-x_{n}\right\|+2 \beta_{n}\left\|x_{n}-y_{n}\right\|\left\|T x_{n}-T p\right\|,
\end{aligned}
$$

By Steps 1-3 and $\lim _{n \rightarrow \infty} \alpha_{n}=0$, it follows from (3.42) that $\lim _{n \rightarrow \infty}\left\|x_{n}-y_{n}\right\|=0$.

Since $\left\|h_{n}-y_{n}\right\| \leq\left\|\gamma A^{*}\right\|\left\|l_{n}-A y_{n}\right\|$ for all $n \in \mathbb{N} \cup\{0\}$, we have $\lim _{n \rightarrow \infty}\left\|h_{n}-y_{n}\right\|=0$. Using it with $\lim _{n \rightarrow \infty}\left\|x_{n}-y_{n}\right\|=0$ and $\lim _{n \rightarrow \infty}\left\|x_{n}-z_{n}\right\|=0$, we get $\lim _{n \rightarrow \infty}\left\|x_{n}-h_{n}\right\|=0$.

Step 5. Prove $\lim _{n \rightarrow \infty}\left\|W_{n} x_{n}-x_{n}\right\|=0$ and $\lim _{n \rightarrow \infty}\left\|W x_{n}-x_{n}\right\|=0$.

Indeed, since

$$
\left\|W_{n} h_{n}-x_{n}\right\|=\frac{1}{\theta}\left\|z_{n}-x_{n}\right\| \longrightarrow 0 \quad \text { as } n \longrightarrow \infty
$$

we have

$$
\left\|W_{n} x_{n}-x_{n}\right\| \leq\left\|W_{n} x_{n}-W_{n} h_{n}\right\|+\left\|W_{n} h_{n}-x_{n}\right\| \leq\left\|x_{n}-h_{n}\right\|+\left\|W_{n} h_{n}-x_{n}\right\| \longrightarrow 0 \quad \text { as } n \longrightarrow \infty \text {. }
$$

By Theorem 2.3, $\lim _{n \rightarrow \infty}\left\|W x_{n}-W_{n} x_{n}\right\|=0$. Since

$$
\left\|W x_{n}-x_{n}\right\| \leq\left\|W x_{n}-W_{n} x_{n}\right\|+\left\|W_{n} x_{n}-x_{n}\right\| \quad \forall n,
$$

we obtain $\lim _{n \rightarrow \infty}\left\|W x_{n}-x_{n}\right\|=0$.

Step 6. There exists a unique $q \in \Omega \subset H$ such that $P_{\Omega} f(q)=q$.

Indeed, for any $x, y \in H$,

$$
\left\|P_{\Omega} f(x)-P_{\Omega} f(y)\right\| \leq\|f(x)-f(y)\| \leq \alpha\|x-y\| .
$$

Since $\alpha \in[0,1), P_{\Omega} f$ is a contraction on $H$. Applying Banach contraction principle, there exists a unique $q \in H$ such that $q=P_{\Omega} f(q) \in \Omega$.

Step 7. Prove $\limsup _{n \rightarrow \infty}\left\langle f q-q, x_{n}-q\right\rangle \leq 0$.

For this purpose, we may choose subsequence $\left\{x_{n_{i}}\right\}$ of $\left\{x_{n}\right\}$ such that

$$
\limsup _{n \rightarrow \infty}\left\langle f q-q, x_{n}-q\right\rangle=\lim _{i \rightarrow \infty}\left\langle f q-q, x_{n_{i}}-q\right\rangle
$$


Since $\left\{x_{n_{i}}\right\}$ is a bounded sequence, there exists a subsequence of $\left\{x_{n_{i}}\right\}$, which is still denoted by $\left\{x_{n_{i}}\right\}$, such that $x_{n_{i}} \rightarrow x^{*} \in H$. Therefore, we have

$$
\limsup _{i \rightarrow \infty}\left\langle f q-q, x_{n_{i}}-q\right\rangle=\left\langle f q-q, x^{*}-q\right\rangle
$$

Next we prove $x^{*} \in \Omega$.

(a) $W x^{*}=x^{*}$. In fact, if $W x^{*} \neq x^{*}$, then we have

$$
\begin{aligned}
\liminf _{i \rightarrow \infty}\left\|x_{n_{i}}-x^{*}\right\| & <\liminf _{i \rightarrow \infty}\left\|x_{n_{i}}-W x^{*}\right\| \\
& \leq \liminf _{i \rightarrow \infty}\left(\left\|x_{n_{i}}-W x_{n_{i}}\right\|+\left\|W x_{n_{i}}-W x^{*}\right\|\right) \\
& \leq \liminf _{i \rightarrow \infty}\left(\left\|x_{n_{i}}-W x_{n_{i}}\right\|+\left\|x_{n_{i}}-x^{*}\right\|\right) \\
& =\liminf _{i \rightarrow \infty}\left\|x_{n_{i}}-x^{*}\right\| \quad \text { (by Step } 5 \text { and Lemma 2.9). }
\end{aligned}
$$

This is a contradiction. Hence, $W x^{*}=x^{*}$, which implies that $x^{*} \in \bigcap_{i=1}^{\infty} F\left(T_{i}\right)$ by Theorem 2.2. Let

(b) Prove $x^{*} \in \operatorname{VI}(C, T)$. Since $x_{n_{i}} \rightarrow x^{*}$ and $\lim _{i \rightarrow \infty}\left\|x_{n_{i}}-y_{n_{i}}\right\|=0$, we have $y_{n_{i}} \rightarrow x^{*}$.

$$
T_{1} x= \begin{cases}T x+N_{C} x, & x \in C, \\ \emptyset, & x \notin C .\end{cases}
$$

Since $T$ is $u$-inversely monotone, $T$ is monotone and hence $T_{1}$ is a maximal monotone mapping. For any given $(x, z) \in G\left(T_{1}\right)$, since $z-T x \in N_{C} x$ and $y_{n} \in C$, by the definition of $N_{C}$, we have

$$
\left\langle x-y_{n}, z-T x\right\rangle \geq 0 \quad \forall n
$$

On the other hand, since $y_{n}=P_{C}\left(I-\beta_{n} T\right) x_{n}$, we have

$$
\left\langle x-y_{n}, y_{n}-\left(x_{n}-\beta_{n} T x_{n}\right)\right\rangle \geq 0
$$

In particular,

$$
\left\langle x-y_{n}, \frac{y_{n}-x_{n}}{\beta_{n}}+T x_{n}\right\rangle \geq 0 \quad \forall n .
$$


From (3.51) and (3.53) we have

$$
\begin{aligned}
\left\langle x-y_{n_{i}}, z\right\rangle & \geq\left\langle x-y_{n_{i}}, T x\right\rangle \\
& \geq\left\langle x-y_{n_{i}}, T x\right\rangle-\left\langle x-y_{n_{i}}, \frac{y_{n_{i}}-x_{n_{i}}}{\beta_{n_{i}}}+T x_{n_{i}}\right\rangle \\
& =\left\langle x-y_{n_{i}}, T x-T y_{n_{i}}\right\rangle+\left\langle x-y_{n_{i}}, T y_{n_{i}}-T x_{n_{i}}\right\rangle-\left\langle x-y_{n_{i}}, \frac{y_{n_{i}}-x_{n_{i}}}{\beta_{n_{i}}}\right\rangle \\
& \geq\left\langle x-y_{n_{i}}, T y_{n_{i}}-T x_{n_{i}}\right\rangle-\left\langle x-y_{n_{i}}, \frac{y_{n_{i}}-x_{n_{i}}}{\beta_{n_{i}}}\right\rangle,
\end{aligned}
$$

which implies

$$
\left\langle x-x^{*}, z\right\rangle=\lim _{i \rightarrow \infty}\left\langle x-y_{n_{i}}, z\right\rangle \geq 0 .
$$

This shows $0 \in T_{1} x^{*}$, that is, $x^{*} \in \operatorname{VI}(C, T)$.

(c) Prove $A x^{*} \in \operatorname{VI}(K, S)$ and $A x^{*} \in F(U)$. Let

$$
T_{2} x= \begin{cases}S x+N_{K} x, & x \in K, \\ \emptyset, & x \notin K .\end{cases}
$$

Then $T_{2}$ is a maximal monotone mapping. For any given $(x, z) \in G\left(T_{2}\right)$, since $z-S x \in N_{K} x$ and $l_{n} \in K$, by the definition of $N_{K}$ we have

$$
\left\langle x-l_{n}, z-S x\right\rangle \geq 0 \quad \forall n .
$$

On the other hand, since $l_{n}=P_{K}\left(I-\beta_{n} S\right) A y_{n}$, we have

$$
\left\langle x-l_{n}, l_{n}-\left(A y_{n}-\beta_{n} S A y_{n}\right)\right\rangle \geq 0,
$$

and hence

$$
\left\langle x-l_{n}, \frac{l_{n}-A y_{n}}{\beta_{n}}+S A y_{n}\right\rangle \geq 0 \quad \forall n .
$$

Since $x_{n_{i}} \rightarrow x^{*}$ and $\lim _{n \rightarrow \infty}\left\|x_{n_{i}}-y_{n_{i}}\right\|=0$ and $\lim _{n \rightarrow \infty}\left\|l_{n_{i}}-A y_{n_{i}}\right\|=0$, we have $l_{n_{i}} \rightarrow A x^{*}$. From (3.57) and (3.59) we have

$$
\begin{aligned}
\left\langle x-l_{n_{i}}, z\right\rangle & \geq\left\langle x-l_{n_{i}}, S x\right\rangle \\
& \geq\left\langle x-l_{n_{i}}, S x-S l_{n_{i}}\right\rangle+\left\langle x-l_{n_{i}}, S l_{n_{i}}-S A y_{n_{i}}\right\rangle-\left\langle x-l_{n_{i}}, \frac{l_{n_{i}}-A y_{n_{i}}}{\beta_{n_{i}}}\right\rangle \\
& \geq\left\langle x-l_{n_{i}}, l_{n_{i}}-A y_{n_{i}}\right\rangle-\left\langle x-l_{n_{i}}, \frac{l_{n_{i}}-A y_{n_{i}}}{\beta_{n_{i}}}\right\rangle .
\end{aligned}
$$


So

$$
\left\langle x-A x^{*}, z\right\rangle=\lim _{i \rightarrow \infty}\left\langle x-l_{n_{i}}, z\right\rangle \geq 0 .
$$

This shows $0 \in T_{2} A x^{*}$, that is, $A x^{*} \in \mathrm{VI}(K, S)$. In addition, by (3.36), (3.37), and Opial's condition, we can prove easily $A x^{*} \in F(U)$.

By (a), (b), and (c), $x^{*} \in \Omega$ is proved. Hence

$$
\limsup _{n \rightarrow \infty}\left\langle f q-q, x_{n}-q\right\rangle=\left\langle f q-q, x^{*}-q\right\rangle \leq 0
$$

Step 8. Prove $\left\{x_{n}\right\}$ converges strongly to $q=P_{\Omega} f(q) \in \Omega$.

In fact, by Step 7 we have

$$
\limsup _{n \rightarrow \infty}\left\langle f q-q, x_{n}-q\right\rangle \leq 0
$$

It follows from (3.5) and Lemma 2.8 that

$$
\begin{aligned}
\left\|x_{n+1}-q\right\|^{2}= & \left\|\alpha_{n} f\left(x_{n}\right)+\left(1-\alpha_{n}\right) z_{n}-q\right\|^{2} \\
\leq & \left(1-\alpha_{n}\right)^{2}\left\|z_{n}-q\right\|^{2}+2 \alpha_{n}\left\langle f\left(x_{n}\right)-q, x_{n+1}-q\right\rangle \\
\leq & \left(1-\alpha_{n}\right)^{2}\left\|x_{n}-q\right\|^{2}+2 \alpha_{n}\left\langle f\left(x_{n}\right)-f(q)+f(q)-q, x_{n+1}-q\right\rangle \\
\leq & \left(1-\alpha_{n}\right)^{2}\left\|x_{n}-q\right\|^{2}+2 \alpha_{n} \alpha\left\|x_{n}-q\right\|\left\|x_{n+1}-q\right\|+2 \alpha_{n}\left\langle f(q)-q, x_{n+1}-q\right\rangle \\
\leq & \left(1-\alpha_{n}(2-2 \alpha)\right)\left\|x_{n}-q\right\|^{2}+\alpha_{n}^{2}\left\|x_{n}-q\right\|^{2}+2 \alpha_{n} \alpha\left\|x_{n}-q\right\|\left\|x_{n+1}-x_{n}\right\| \\
& +2 \alpha_{n}\left\langle f(q)-q, x_{n+1}-q\right\rangle \quad \forall n \in \mathbb{N} \cup\{0\} .
\end{aligned}
$$

Let $\lambda_{n}=\alpha_{n}(2-2 \alpha), n \in \mathbb{N} \cup\{0\}$. Then, for any $n \in \mathbb{N} \cup\{0\}$, we obtain

$$
\begin{gathered}
\alpha_{n}^{2}\left\|x_{n}-q\right\|^{2}=\frac{\lambda_{n} \alpha_{n}}{2-2 \alpha}\left\|x_{n}-q\right\|^{2}, \\
2 \alpha_{n} \alpha\left\|x_{n}-q\right\|\left\|x_{n+1}-x_{n}\right\|=\frac{\lambda_{n} \alpha}{1-\alpha}\left\|x_{n}-q\right\|\left\|x_{n+1}-x_{n}\right\|, \\
2 \alpha_{n}\left\langle f(q)-q, x_{n+1}-q\right\rangle=\frac{\lambda_{n}}{1-\alpha}\left\langle f(q)-q, x_{n+1}-q\right\rangle .
\end{gathered}
$$

Set

$$
b_{n}=\frac{\alpha_{n}}{2-2 \alpha}\left\|x_{n}-q\right\|^{2}+\frac{\alpha}{1-\alpha}\left\|x_{n}-q\right\|\left\|x_{n+1}-x_{n}\right\|+\frac{1}{1-\alpha}\left\langle f(q)-q, x_{n+1}-q\right\rangle .
$$


The condition (C1) and the boundedness of $\left\{x_{n}\right\}$ ensure $\lim _{n \rightarrow \infty}\left(\alpha_{n} /(2-2 \alpha)\right)\left\|x_{n}-q\right\|^{2}=0$, Step 2 ensure $\lim _{n \rightarrow \infty}(1 /(1-\alpha)) \alpha\left\|x_{n}-q\right\|\left\|x_{n+1}-x_{n}\right\|=0$, and Step 7 ensure lim $\sup _{n \rightarrow \infty}(1 /(1-$ $\alpha))\left\langle f(q)-q, x_{n+1}-q\right\rangle \leq 0$, so $\lim \sup _{n \rightarrow \infty} b_{n} \leq 0$. Applying Lemma 2.6 and the inequality

$$
\left\|x_{n+1}-q\right\|^{2} \leq\left(1-\lambda_{n}\right)\left\|x_{n}-q\right\|^{2}+\lambda_{n} b_{n}
$$

we obtain that $\lim _{n \rightarrow \infty}\left\|x_{n}-q\right\|=0$ which means that the sequence $\left\{x_{n}\right\}$ strongly converge to q. This completes the proof of the Theorem 3.5. $U=I$.

The following convergence theorems can be established by applying Theorem 3.5 with

Corollary 3.6. Let $H_{1}, H_{2}$ be two real Hilbert spaces and $C \subset H_{1}, K \subset H_{2}$ two nonempty closed convex sets. Let $T: C \rightarrow H_{1}$ and $S: K \rightarrow H_{2}$ be u-inversely monotone. $A: H_{1} \rightarrow H_{2}$ is a bounded linear operator with adjoint operator $A^{*}$. Let $f: C \rightarrow C$ be a contraction with contraction constant $\alpha$. Let $\left\{T_{i}\right\}_{i \in \mathbb{N}}$ be a family of infinitely nonexpansive mappings of $C$ into itself such that $\Omega=\left\{p \in \bigcap_{i=1}^{\infty} F\left(T_{i}\right) \cap \operatorname{VI}(C, T): A p \in \operatorname{VI}(K, S)\right\} \neq \emptyset$. Let $\xi$ be a real number and $\left\{\lambda_{i}\right\}_{i \in \mathbb{N}}$ be a sequence of real numbers such that $0<\lambda_{i} \leq \xi<1$, for every $i \in \mathbb{N}$. For each $n \in \mathbb{N}$, let $W_{n}$ be the $W$-mapping of $C$ into itself generated by $T_{n}, T_{n-1}, \ldots, T_{1}$ and $\lambda_{n}, \lambda_{n-1}, \ldots, \lambda_{1}$. Let $\left\{x_{n}\right\}$ be a sequence generated by the following algorithm:

$$
\begin{gathered}
x_{0}=x \in C \quad \text { chosen arbitrarily, } \\
y_{n}=P_{C}\left(I-\beta_{n} T\right) x_{n}, \\
l_{n}=P_{K}\left(I-\beta_{n} S\right) A y_{n}, \\
z_{n}=(1-\theta) x_{n}+\theta W_{n} P_{C}\left(y_{n}+r A^{*}\left(l_{n}-A y_{n}\right)\right), \\
x_{n+1}=\alpha_{n} f\left(x_{n}\right)+\left(1-\alpha_{n}\right) z_{n}, \quad \forall n \in \mathbb{N} \cup\{0\},
\end{gathered}
$$

where $r \in\left(0,1 /\|A\|^{2}\right)$ and $\theta \in(0,1)$ are two constants and $\left\{\alpha_{n}\right\}_{n=0}^{\infty}$ and $\left\{\beta_{n}\right\}_{n=0}^{\infty}$ are two sequences in $(0,1)$. If $\left\{\alpha_{n}\right\}_{n=0}^{\infty}$ and $\left\{\beta_{n}\right\}_{n=0}^{\infty}$ further satisfy the following conditions:

$\left(C_{1}\right) \lim _{n \rightarrow \infty} \alpha_{n}=0$ and $\sum_{n=1}^{\infty} \alpha_{n}=\infty$;

$\left(C_{2}\right)\left\{\beta_{n}\right\} \subset[a, b]$ and $\lim _{n \rightarrow \infty}\left|\beta_{n+1}-\beta_{n}\right|=0$, where $0<a, b<2 u$;

then the following statements hold:

(a) there exists a unique $q \in \Omega$ such that $P_{\Omega} f(q)=q$;

(b) $\left\{x_{n}\right\}$ converges strongly to $q$.

If $T_{i}=I$ for all $i \in \mathbb{N}$ in Theorem 3.5, then we have the following result.

Corollary 3.7. Let $H_{1}, H_{2}$ be two real Hilbert spaces and $\mathrm{C} \subset H_{1}, K \subset H_{2}$ two nonempty closed convex sets. Let $T: C \rightarrow H_{1}$ and $S: K \rightarrow H_{2}$ be u-inversely monotone. $A: H_{1} \rightarrow H_{2}$ is a bounded linear operator with adjoint operator $A^{*}$. Let $f: C \rightarrow C$ be a contraction with contraction constant $\alpha$. Let $U$ be a nonexpansive mapping of $K$ into itself such that $\Omega=\{p \in \operatorname{VI}(C, T): A p \in$ $F(U) \cap \operatorname{VI}(K, S)\} \neq \emptyset$. Let $\xi$ be a real number and $\left\{\lambda_{i}\right\}_{i \in \mathbb{N}}$ be a sequence of real numbers such that 
$0<\lambda_{i} \leq \xi<1$, for every $i \in \mathbb{N}$. For each $n \in \mathbb{N}$, let $W_{n}$ be the $W$-mapping of $C$ into itself generated by $T_{n}, T_{n-1}, \ldots, T_{1}$ and $\lambda_{n}, \lambda_{n-1}, \ldots, \lambda_{1}$. Let $\left\{x_{n}\right\}$ be a sequence generated by the following algorithm:

$$
\begin{gathered}
x_{0}=x \in C \quad \text { chosen arbitrarily, } \\
y_{n}=P_{C}\left(I-\beta_{n} T\right) x_{n}, \\
l_{n}=P_{K}\left(I-\beta_{n} S\right) A y_{n}, \\
z_{n}=(1-\theta) x_{n}+\theta P_{C}\left(y_{n}+r A^{*}\left(U l_{n}-A y_{n}\right)\right), \\
x_{n+1}=\alpha_{n} f\left(x_{n}\right)+\left(1-\alpha_{n}\right) z_{n}, \quad \forall n \in \mathbb{N} \cup\{0\},
\end{gathered}
$$

where $r \in\left(0,1 /\|A\|^{2}\right)$ and $\theta \in(0,1)$ are two constants and $\left\{\alpha_{n}\right\}_{n=0}^{\infty}$ and $\left\{\beta_{n}\right\}_{n=0}^{\infty}$ are two sequences in $(0,1)$. If $\left\{\alpha_{n}\right\}_{n=0}^{\infty}$ and $\left\{\beta_{n}\right\}_{n=0}^{\infty}$ further satisfy the following conditions:

$\left(C_{1}\right) \lim _{n \rightarrow \infty} \alpha_{n}=0$ and $\sum_{n=1}^{\infty} \alpha_{n}=\infty$;

$\left(C_{2}\right)\left\{\beta_{n}\right\} \subset[a, b]$ and $\lim _{n \rightarrow \infty}\left|\beta_{n+1}-\beta_{n}\right|=0$, where $0<a, b<2 u$;

then the following statements hold:

(a) there exists a unique $q \in \Omega$ such that $P_{\Omega} f(q)=q$;

(b) $\left\{x_{n}\right\}$ converges strongly to $q$.

In Theorem 3.5, if $T_{i}=I$ for all $i \in \mathbb{N}$ and $U=I$, then we obtain Corollary 3.8.

Corollary 3.8. Let $H_{1}, H_{2}$ be two real Hilbert spaces and $\mathrm{C} \subset H_{1}, K \subset H_{2}$ two nonempty closed convex sets. Let $T: C \rightarrow H_{1}$ and $S: K \rightarrow H_{2}$ be u-inversely monotone. $A: H_{1} \rightarrow H_{2}$ is a bounded linear operator with adjoint operator $A^{*}$. Let $f: C \rightarrow C$ be a contraction with contraction constant $\alpha$. Let $\left\{T_{i}\right\}_{i \in \mathbb{N}}$ be a family of infinitely nonexpansive mappings of $C$ into itself and $U$ a nonexpansive mapping of $K$ into itself such that $\Omega=\{p \in \mathrm{VI}(C, T): A p \in \mathrm{VI}(K, S)\} \neq \emptyset$. Let $\xi$ be a real number and $\left\{\lambda_{i}\right\}_{i \in \mathbb{N}}$ be a sequence of real numbers such that $0<\lambda_{i} \leq \xi<1$, for every $i \in \mathbb{N}$. For each $n \in \mathbb{N}$, let $W_{n}$ be the $W$-mapping of $C$ into itself generated by $T_{n}, T_{n-1}, \ldots, T_{1}$ and $\lambda_{n}, \lambda_{n-1}, \ldots, \lambda_{1}$. Let $\left\{x_{n}\right\}$ be a sequence generated by the following algorithm:

$$
\begin{gathered}
x_{0}=x \in C \quad \text { chosen arbitrarily, } \\
y_{n}=P_{C}\left(I-\beta_{n} T\right) x_{n}, \\
l_{n}=P_{K}\left(I-\beta_{n} S\right) A y_{n}, \\
z_{n}=(1-\theta) x_{n}+\theta P_{C}\left(y_{n}+r A^{*}\left(l_{n}-A y_{n}\right)\right), \\
x_{n+1}=\alpha_{n} f\left(x_{n}\right)+\left(1-\alpha_{n}\right) z_{n}, \quad \forall n \in \mathbb{N} \cup\{0\},
\end{gathered}
$$

where $r \in\left(0,1 /\|A\|^{2}\right)$ and $\theta \in(0,1)$ are two constants and $\left\{\alpha_{n}\right\}_{n=0}^{\infty}$ and $\left\{\beta_{n}\right\}_{n=0}^{\infty}$ are two sequences in $(0,1)$. If $\left\{\alpha_{n}\right\}_{n=0}^{\infty}$ and $\left\{\beta_{n}\right\}_{n=0}^{\infty}$ further satisfy the following conditions:

$\left(C_{1}\right) \lim _{n \rightarrow \infty} \alpha_{n}=0$ and $\sum_{n=1}^{\infty} \alpha_{n}=\infty$;

$\left(C_{2}\right)\left\{\beta_{n}\right\} \subset[a, b]$ and $\lim _{n \rightarrow \infty}\left|\beta_{n+1}-\beta_{n}\right|=0$, where $0<a, b<2 u$; 
then the following statements hold:

(a) there exists a unique $q \in \Omega$ such that $P_{\Omega} f(q)=q$;

(b) $\left\{x_{n}\right\}$ converges strongly to $q$.

In Theorem 3.5, if $H_{1}=H_{2}, C=K$ and $A=A^{*}=I$, then we have Corollary 3.9.

Corollary 3.9. Let $H$ be a real Hilbert space and $C \subset H$ a nonempty closed convex set. Let $T: C \rightarrow$ $H$ and $S: C \rightarrow H$ be u-inversely monotone. Let $f: C \rightarrow C$ be a contraction with contraction constant $\alpha$. Let $U: C \rightarrow C$ be a nonexpansive mapping and $\left\{T_{i}\right\}_{i \in \mathbb{N}}: C \rightarrow C$ a family of infinitely nonexpansive mappings such that $\Omega=\bigcap_{i=1}^{\infty} F\left(T_{i}\right) \cap F(U) \cap \mathrm{VI}(C, T) \cap \mathrm{VI}(K, S) \neq \emptyset$. Let $\xi$ be a real number and $\left\{\lambda_{i}\right\}_{i \in \mathbb{N}}$ be a sequence of real numbers such that $0<\lambda_{i} \leq \xi<1$, for every $i \in \mathbb{N}$. For each $n \in \mathbb{N}$, let $W_{n}$ be the $W$-mapping of $C$ into itself generated by $T_{n}, T_{n-1}, \ldots, T_{1}$ and $\lambda_{n}, \lambda_{n-1}, \ldots, \lambda_{1}$. Let $\left\{x_{n}\right\}$ be a sequence generated by the following algorithm:

$$
\begin{gathered}
x_{0}=x \in C \quad \text { chosen arbitrarily, } \\
y_{n}=P_{C}\left(I-\beta_{n} T\right) x_{n}, \\
l_{n}=P_{C}\left(I-\beta_{n} S\right) y_{n}, \\
z_{n}=(1-\theta) x_{n}+\theta W_{n}\left(y_{n}+r\left(U l_{n}-y_{n}\right)\right), \\
x_{n+1}=\alpha_{n} f\left(x_{n}\right)+\left(1-\alpha_{n}\right) z_{n}, \quad \forall n \in \mathbb{N} \cup\{0\},
\end{gathered}
$$

where $r \in(0,1)$ and $\theta \in(0,1)$ are two constants and $\left\{\alpha_{n}\right\}_{n=0}^{\infty}$ and $\left\{\beta_{n}\right\}_{n=0}^{\infty}$ are two sequences in $(0,1)$. If $\left\{\alpha_{n}\right\}_{n=0}^{\infty}$ and $\left\{\beta_{n}\right\}_{n=0}^{\infty}$ further satisfy the following conditions:

$\left(C_{1}\right) \lim _{n \rightarrow \infty} \alpha_{n}=0$ and $\sum_{n=1}^{\infty} \alpha_{n}=\infty$;

$\left(C_{2}\right)\left\{\beta_{n}\right\} \subset[a, b]$ and $\lim _{n \rightarrow \infty}\left|\beta_{n+1}-\beta_{n}\right|=0$, where $0<a, b<2 u$;

then the following statements hold:

(a) there exists a unique $q \in \Omega$ such that $P_{\Omega} f(q)=q$;

(b) $\left\{x_{n}\right\}$ converges strongly to $q$. result.

In Theorem 3.5, if $H_{1}=H_{2}, C=K A=A^{*}=I$, and $U=I$, then we get the following

Corollary 3.10. Let $H$ be a real Hilbert space and $C \subset H$ a nonempty closed convex set. Let $T: C \rightarrow H$ and $S: C \rightarrow H$ be u-inversely monotone. Let $f: C \rightarrow C$ be a contraction with contraction constant $\alpha$. Let $\left\{T_{i}\right\}_{i \in \mathbb{N}}: C \rightarrow C$ be a family of infinitely nonexpansive mappings such that $\Omega=\bigcap_{i=1}^{\infty} F\left(T_{i}\right) \cap \operatorname{VI}(C, T) \cap \operatorname{VI}(K, S) \neq \emptyset$. Let $\xi$ be a real number and $\left\{\lambda_{i}\right\}_{i \in \mathbb{N}}$ be a sequence of real numbers such that $0<\lambda_{i} \leq \xi<1$, for every $i \in \mathbb{N}$. For each $n \in \mathbb{N}$, let $W_{n}$ be the $W$-mapping of $C$ into itself generated by $T_{n}, T_{n-1}, \ldots, T_{1}$ and $\lambda_{n}, \lambda_{n-1}, \ldots, \lambda_{1}$. Let $\left\{x_{n}\right\}$ be a sequence generated by the following algorithm:

$$
\begin{gathered}
x_{0}=x \in C \quad \text { chosen arbitrarily, } \\
y_{n}=P_{C}\left(I-\beta_{n} T\right) x_{n}, \\
l_{n}=P_{C}\left(I-\beta_{n} S\right) y_{n}, \\
z_{n}=(1-\theta) x_{n}+\theta W_{n}\left(y_{n}+r\left(l_{n}-y_{n}\right)\right), \\
x_{n+1}=\alpha_{n} f\left(x_{n}\right)+\left(1-\alpha_{n}\right) z_{n}, \quad \forall n \in \mathbb{N} \cup\{0\},
\end{gathered}
$$


where $r \in(0,1)$ and $\theta \in(0,1)$ are two constants and $\left\{\alpha_{n}\right\}_{n=0}^{\infty}$ and $\left\{\beta_{n}\right\}_{n=0}^{\infty}$ are two sequences in $(0,1)$. If $\left\{\alpha_{n}\right\}_{n=0}^{\infty}$ and $\left\{\beta_{n}\right\}_{n=0}^{\infty}$ further satisfy the following conditions:

$\left(C_{1}\right) \lim _{n \rightarrow \infty} \alpha_{n}=0$ and $\sum_{n=1}^{\infty} \alpha_{n}=\infty$;

$\left(C_{2}\right)\left\{\beta_{n}\right\} \subset[a, b]$ and $\lim _{n \rightarrow \infty}\left|\beta_{n+1}-\beta_{n}\right|=0$, where $0<a, b<2 u$;

then the following statements hold:

(a) there exists a unique $q \in \Omega$ such that $P_{\Omega} f(q)=q$;

(b) $\left\{x_{n}\right\}$ converges strongly to $q$.

Finally, if we let $H_{1}=H_{2}, C=K A=A^{*}=I, T_{i}=I$, for all $i \in \mathbb{N}$ and $U=I$ in Theorem 3.5, then the following result can be established.

Corollary 3.11. Let $H$ be a real Hilbert space and $C \subset H$ a nonempty closed convex set. Let $T: C \rightarrow$ $H$ and $S: C \rightarrow H$ be u-inversely monotone. Let $f: C \rightarrow C$ be a contraction with contraction constant $\alpha$. Suppose that $\Omega=\operatorname{VI}(C, T) \cap \operatorname{VI}(K, S) \neq \emptyset$. Let $\left\{x_{n}\right\}$ be a sequence generated by the following algorithm:

$$
\begin{gathered}
x_{0}=x \in C \quad \text { chosen arbitrarily, } \\
y_{n}=P_{C}\left(I-\beta_{n} T\right) x_{n}, \\
l_{n}=P_{C}\left(I-\beta_{n} S\right) y_{n}, \\
z_{n}=(1-\theta) x_{n}+\theta\left(y_{n}+r\left(l_{n}-y_{n}\right)\right), \\
x_{n+1}=\alpha_{n} f\left(x_{n}\right)+\left(1-\alpha_{n}\right) z_{n}, \quad \forall n \in \mathbb{N} \cup\{0\},
\end{gathered}
$$

where $r \in(0,1)$ and $\theta \in(0,1)$ are two constants and $\left\{\alpha_{n}\right\}_{n=0}^{\infty}$ and $\left\{\beta_{n}\right\}_{n=0}^{\infty}$ are two sequences in $(0,1)$. If $\left\{\alpha_{n}\right\}_{n=0}^{\infty}$ and $\left\{\beta_{n}\right\}_{n=0}^{\infty}$ further satisfy the following conditions:

$\left(C_{1}\right) \lim _{n \rightarrow \infty} \alpha_{n}=0$ and $\sum_{n=1}^{\infty} \alpha_{n}=\infty$;

$\left(C_{2}\right)\left\{\beta_{n}\right\} \subset[a, b]$ and $\lim _{n \rightarrow \infty}\left|\beta_{n+1}-\beta_{n}\right|=0$, where $0<a, b<2 u$;

then the following statements hold:

(a) there exists a unique $q \in \Omega$ such that $P_{\Omega} f(q)=q$;

(b) $\left\{x_{n}\right\}$ converges strongly to $q$.

Remark 3.12. (a) In $[11,16]$, the authors gave some algorithms for $(u, v)$-cocoercive and $\mu$-Lipschitz continuous operator and obtain some strongly convergence theorems; see [11, Theorems 2.1 and 2.2] and [16, Corollary 3.3]. However, the $(u, v)$-cocoercive and $\mu$-Lipschitz continuous operator considered by $[11,16]$ is actually a strongly monotone and $\mu$-Lipschitz continuous operator. Then, by Remark ST, such operators studied in [11, 16] are $u$-inverse strongly monotone. Hence our results obtained in this paper conclude some results in $[11,16]$ as special cases.

(b) Our results are different from the main results in $[9-11,16]$ and references therein.

\section{Acknowledgments}

The first author was supported by the Natural Science Foundation of Yunnan Province (2010ZC152) and the Scientific Research Foundation from Yunnan Province Education 
Committee (08Y0338). The second author was supported partially by Grant no. NSC 1012115-M-017-001 of the National Science Council of China.

\section{References}

[1] N. X. Tan, "Quasivariational inequalities in topological linear locally convex Hausdorff spaces," Mathematische Nachrichten, vol. 122, pp. 231-245, 1985.

[2] E. Blum and W. Oettli, "From optimization and variational inequalities to equilibrium problems," The Mathematics Student, vol. 63, no. 1-4, pp. 123-145, 1994.

[3] P. Deguire, K. K. Tan, and G. X.-Z. Yuan, "The study of maximal elements, fixed points for $L_{S^{-}}$ majorized mappings and their applications to minimax and variational inequalities in product topological spaces," Nonlinear Analysis A, vol. 37, no. 7, pp. 933-951, 1999.

[4] G. Isac, V. A. Bulavsky, and V. V. Kalashnikov, Complementarity, Equilibrium, Efficiency and Economics, Kluwer, Dordrecht, The Netherlands, 2002.

[5] Q. H. Ansari, L. J. Lin, and L. B. Su, "Systems of simultaneous generalized vector quasiequilibrium problems and their applications," Journal of Optimization Theory and Applications, vol. 127, no. 1, pp. 27-44, 2005.

[6] L.-J. Lin and W.-S. Du, "Systems of equilibrium problems with applications to new variants of Ekeland's variational principle, fixed point theorems and parametric optimization problems," Journal of Global Optimization, vol. 40, no. 4, pp. 663-677, 2008.

[7] Y. Censor, A. Gibali, S. Reich, and S. Sabach, "Common solutions to variational inequalities," SetValued and Variational Analysis, vol. 20, no. 2, pp. 229-247, 2012.

[8] Y. Censor, A. Gibali, and S. Reich, "Algorithms for the split variational inequality problem," Numerical Algorithms, vol. 59, no. 2, pp. 301-323, 2012.

[9] H. Iiduka and W. Takahashi, "Strong convergence theorems for nonexpansive mappings and inversestrongly monotone mappings," Nonlinear Analysis A, vol. 61, no. 3, pp. 341-350, 2005.

[10] J. Chen, L. Zhang, and T. Fan, "Viscosity approximation methods for nonexpansive mappings and monotone mappings," Journal of Mathematical Analysis and Applications, vol. 334, no. 2, pp. 1450-1461, 2007.

[11] Z. Huang and M. A. Noor, "Some new unified iteration schemes with errors for nonexpansive mappings and variational inequalities," Applied Mathematics and Computation, vol. 194, no. 1, pp. 135142, 2007.

[12] M. Aslam Noor, "Some developments in general variational inequalities," Applied Mathematics and Computation, vol. 152, no. 1, pp. 199-277, 2004.

[13] R. E. Bruck, Jr., "On the weak convergence of an ergodic iteration for the solution of variational inequalities for monotone operators in Hilbert space," Journal of Mathematical Analysis and Applications, vol. 61, no. 1, pp. 159-164, 1977.

[14] Y. Yao and J.-C. Yao, "On modified iterative method for nonexpansive mappings and monotone mappings," Applied Mathematics and Computation, vol. 186, no. 2, pp. 1551-1558, 2007.

[15] B. He, L.-Z. Liao, D. Han, and H. Yang, "A new inexact alternating directions method for monotone variational inequalities," Mathematical Programming, vol. 92, no. 1, pp. 103-118, 2002.

[16] J.-W. Peng, S.-Y. Wu, and J.-C. Yao, "A new iterative method for finding common solutions of a system of equilibrium problems, fixed-point problems, and variational inequalities," Abstract and Applied Analysis, vol. 2010, Article ID 428293, 27 pages, 2010.

[17] Z. He, "The split equilibrium problem and its convergence algorithms," Journal of Inequalities and Applications, vol. 2012, p. 162, 2012.

[18] Z. He and W.-S. Du, "Nonlinear algorithms approach to split common solution problems," Fixed Point Theory and Applications, vol. 2012, p. 130, 2012.

[19] W. Takahashi, Nonlinear Functional Analysis, Fixed Point Theory and Its Applications, Yokohama Publishers, Yokohama, Japan, 2000.

[20] R. T. Rockafellar, "Monotone operators and the proximal point algorithm," SIAM Journal on Control and Optimization, vol. 14, no. 5, pp. 877-898, 1976.

[21] R. T. Rockafellar, "On the maximality of sums of nonlinear monotone operators," Transactions of the American Mathematical Society, vol. 149, pp. 75-88, 1970. 
[22] K. Shimoji and W. Takahashi, "Strong convergence to common fixed points of infinite nonexpansive mappings and applications," Taiwanese Journal of Mathematics, vol. 5, no. 2, pp. 387-404, 2001.

[23] S.-s. Chang, H. W. Joseph Lee, and C. K. Chan, "A new method for solving equilibrium problem fixed point problem and variational inequality problem with application to optimization," Nonlinear Analysis A, vol. 70, no. 9, pp. 3307-3319, 2009.

[24] H. K. Xu, "An iterative approach to quadratic optimization," Journal of Optimization Theory and Applications, vol. 116, no. 3, pp. 659-678, 2003.

[25] T. Suzuki, "Strong convergence theorems for infinite families of nonexpansive mappings in general Banach spaces," Fixed Point Theory and Applications, no. 1, pp. 103-123, 2005.

[26] S. S. Chang, "Some problems and results in the study of nonlinear analysis," Nonlinear Analysis A, vol. 30, pp. 4197-4208, 1997. 


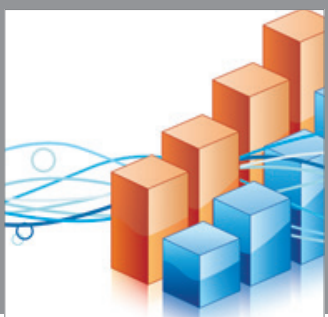

Advances in

Operations Research

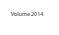

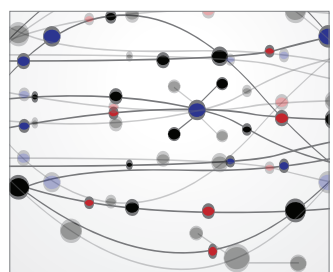

\section{The Scientific} World Journal
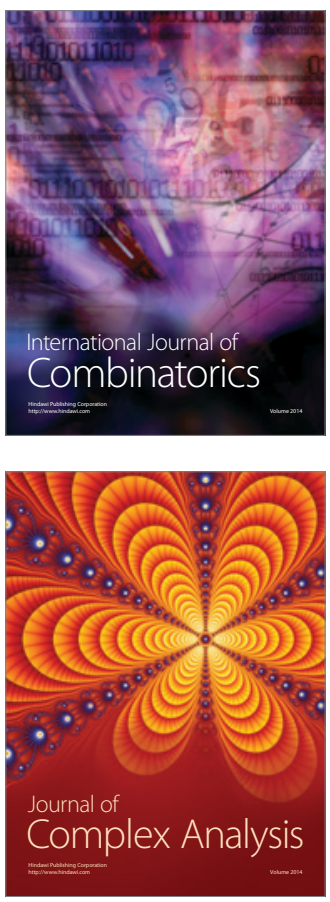

International Journal of

Mathematics and

Mathematical

Sciences
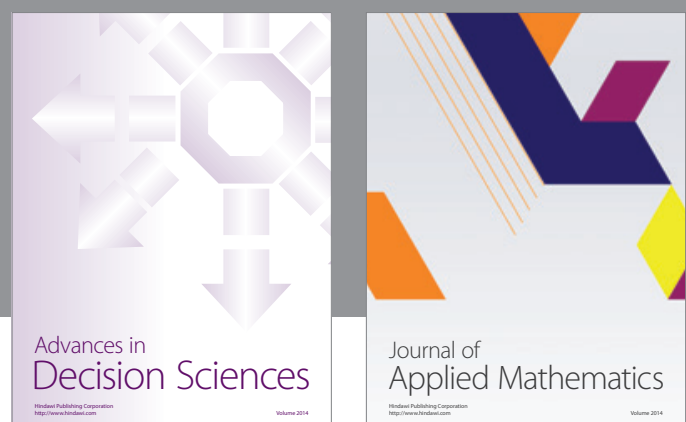

Journal of

Applied Mathematics
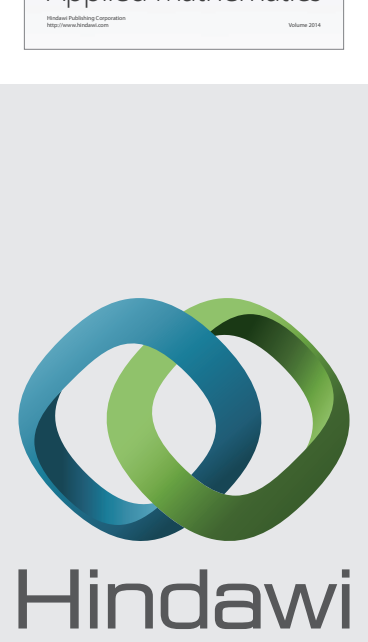

Submit your manuscripts at http://www.hindawi.com
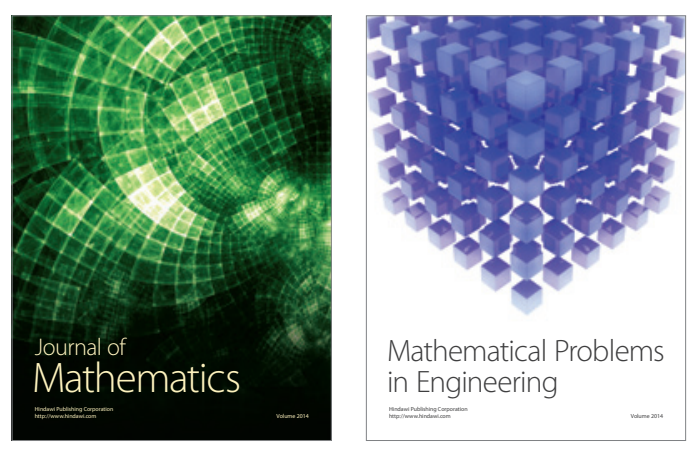

Mathematical Problems in Engineering
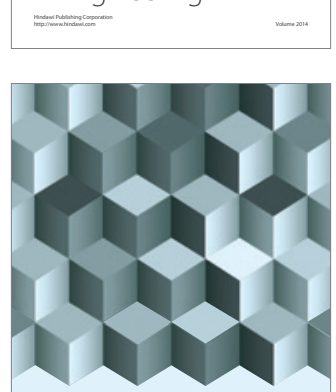

Journal of

Function Spaces
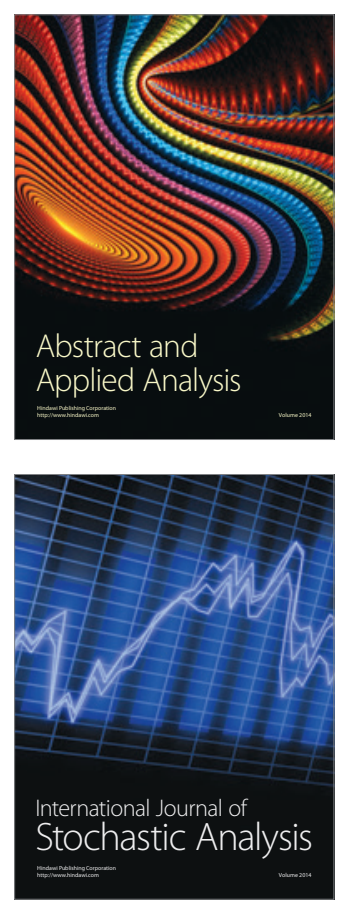

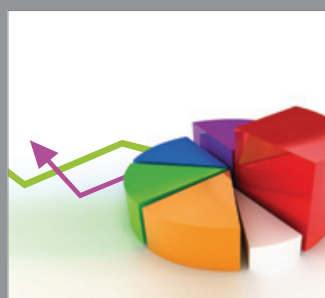

ournal of

Probability and Statistics

Promensencen
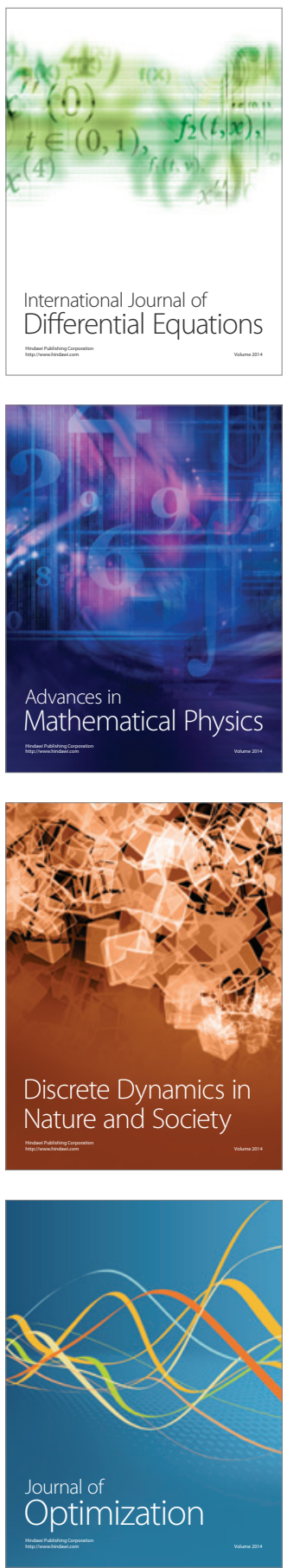